\title{
Balanced Optical Phase Diversity Receivers for Coherence Multiplexing
}

\author{
Arjan Meijerink, Student Member, IEEE, Geert H. L. M. Heideman, and Wim van Etten, Senior Member, IEEE
}

\begin{abstract}
Coherence multiplexing (CM) is a specific form of CDMA that is particularly suitable for optical communication systems for medium data rates and small distances. By virtue of its potentially simple implementation, it is particularly useful for "bridging the last mile" to the subscribers, which is an issue that is receiving considerable attention in optical communication research nowadays. The receivers in coherence multiplex systems published so far were all based on either self-homodyne or self-heterodyne detection. Both solutions are rather complicated, and therefore, expensive. In this paper, a new $\mathrm{CM}$ receiver concept is proposed, based on optical phase diversity detection. Theoretical performance results are derived for several modulation formats and receiver structures.
\end{abstract}

Index Terms-Access techniques, coherence multiplexing, optical CDMA, optical communication, phase diversity.

\section{INTRODUCTION}

C OHERENCE MULTIPLEXING (CM) is a specific optical multiplex technique, in which the temporal coherence of broadband light sources is being utilized in order to distinguish between channels. It can be categorized as a photonic code division multiplexing scheme [1], as it shares the following properties with the well-known direct-sequence optical coding techniques:

1) asynchronous operation in the same wavelength band;

2) low access delay;

3) coding and decoding performed in the optical domain;

4) "soft capacity": the performance gracefully degrades with increasing number of channels.

The particular advantages of CM are that broadband sources and very simple selection components are required-which enables cheap implementation-and that the scheme is very robust to crosstalk due to for example temperature drift or aging of components. Moreover, optical modulation is performed at a rate that corresponds to the data rate, rather than the considerably higher chip rates that are used in direct sequence techniques. Drawbacks are the limited number of channels and data rates due to multiple access interference, and the limited span

Manuscript received December 12, 2003; revised June 17, 2004. The work of A. Meijerink was supported by Philips Research, Eindhoven, The Netherlands.

A. Meijerink and W. van Etten are with the Telecommunication Engineering Group, Faculty of Electrical Engineering, Mathematics and Computer Science, University of Twente, $7500 \mathrm{AE}$, Enschede, The Netherlands (e-mail: a.meijerink@ieee.org).

G. H. L. M. Heideman, retired, was with the Telecommunication Engineering Group, Faculty of Electrical Engineering, Mathematics and Computer Science, University of Twente, $7500 \mathrm{AE}$, Enschede, The Netherlands.

Digital Object Identifier 10.1109/JLT.2004.834502 lengths, since the large linewidths of the sources renders the information transmission rather sensitive to chromatic fiber dispersion. Therefore, CM should not be considered as a way of enhancing the bandwidth utilization in long haul fiber networks, but rather as an inexpensive means of performing multiple access on smaller-scale networks in for instance the access or local area.

All previously published CM receivers are based either on self-homodyne or self-heterodyne detection. In this paper, it will be shown theoretically that a stable output signal can be obtained by applying a phase diversity network in the receiver. Its main benefit is that neither a phase synchronization scheme nor an optical frequency shifter is required.

In the next section, the basic principle of CM will be summarized. After that, a more detailed description of the phase sensitivity issue will be given, together with a discussion of its known solutions, namely phase synchronization and frequency shifting. Then, it will be explained how the output signal of a $\mathrm{CM}$ receiver can be stabilized by applying a phase diversity network. Before coming to conclusions, an analysis is presented in which the bit error probabilities of various receiver types are derived and compared.

\section{BASIC PRINCIPLE OF COHERENCE MUlTiPleXING (CM)}

\section{A. Channel Generation and Selection}

Consider the simplified CM system in Fig. 1, consisting of only one transmitter and one receiver. The transmitter consists of a broadband light source and a two-arm Mach-Zehnder interferometer (MZI). The light that is coupled into the waveguide is assumed to be linearly polarized light, having an electrical field with pre-envelope $x(t)$, which is normalized such that the average power that is launched into the MZI is given by $P_{\text {in }}=(1 / 2) \mathrm{E}\left[|x(t)|^{2}\right]$, where E[.] denotes expected value. (As a result, this notation does not provide information about the actual mode profile of the electrical field.) The MZI comprises two $2 \times 2$ directional couplers, having transfer matrix

$$
\left[H_{2}\right]=\frac{1}{\sqrt{2}}\left[\begin{array}{ll}
1 & j \\
j & 1
\end{array}\right] \text {. }
$$

The path imbalance (i.e., the difference in propagation delay of the two interferometer paths) is denoted by $T_{\mathrm{Tx}}$. (For simplicity, it is assumed that this denotes both the phase delay as well as the group delay.) Two different modulation methods are considered: phase modulation (PM) and intensity modulation (IM). Phase modulation is performed in the lower arm of the MZI and is denoted by $\phi_{\bmod }(t)$, which is a real signal with a value between 


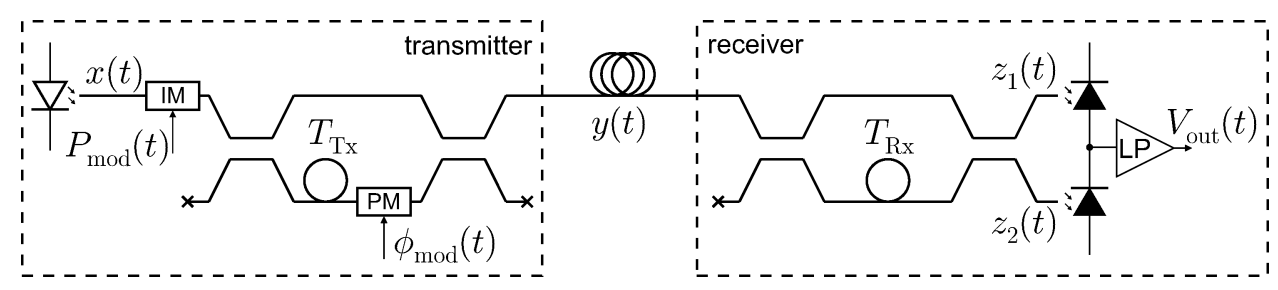

Fig. 1. Conventional coherence multiplex system. For simplicity, only one transmitter and one (balanced) receiver are shown, to illustrate the CM channel generation and selection concept. Both phase modulation (PM) and intensity modulation (IM) are displayed, to enable a general analysis which does not depend on the modulation format. (In practice only one modulator will be there.)

0 and $2 \pi$ radians. Intensity modulation is performed directly on the source signal, and is denoted by $P_{\bmod }(t)$, which is a real signal between 0 and 1 (without unit). In practice, only one of the modulators is used, but here it is temporarily assumed that both of them are there, in order to facilitate a general analysis of the $\mathrm{CM}$ channel generation and selection concept, independent of the modulation format. We will come back to this at the end of this subsection, where two specific modulation formats are considered. Although $\phi_{\text {mod }}(t)$ and $P_{\text {mod }}(t)$ actually represent information signals, they will for the time being be considered as (known) deterministic signals. Hence, when insertion losses, coupling losses and propagation losses are neglected, and the mutual polarization of the light waves is assumed to be maintained, the total electrical field in the transmission fiber is a nonstationary random process with a pre-envelope denoted by

$$
y(t)=\frac{1}{2} \sqrt{P_{\bmod }(t)}\left[x(t)-x\left(t-T_{\mathrm{Tx}}\right) \exp \left(j \phi_{\bmod }(t)\right)\right]
$$

where it is assumed that changes in $P_{\bmod }(t)$ in an interval of length $T_{\mathrm{Tx}}$ are negligible, i.e., the bandwidth of $P_{\bmod }(t)$ is assumed to be much smaller than the inverse of $T_{\mathrm{Tx}}$. The autocorrelation function of $y(t)$ is given by

$$
\begin{aligned}
R_{y^{*} y}\left(t_{1}, t_{2}\right) \triangleq & \mathrm{E}\left[y^{*}\left(t_{1}\right) y\left(t_{2}\right)\right] \\
=\frac{1}{4} & \sqrt{P_{\bmod }\left(t_{1}\right) P_{\bmod }\left(t_{2}\right)} \\
\cdot[ & R_{x^{*} x}\left(t_{2}-t_{1}\right) \\
& \quad-R_{x^{*} x}\left(t_{2}-t_{1}-T_{\mathrm{Tx}}\right) \exp \left(j \phi_{\bmod }\left(t_{2}\right)\right) \\
& \quad-R_{x^{*} x}\left(t_{2}-t_{1}+T_{\mathrm{Tx}}\right) \exp \left(-j \phi_{\bmod }\left(t_{1}\right)\right) \\
& +R_{x^{*} x}\left(t_{2}-t_{1}\right) \\
& \left.\cdot \exp \left(j\left(\phi_{\bmod }\left(t_{2}\right)-\phi_{\bmod }\left(t_{1}\right)\right)\right)\right]
\end{aligned}
$$

where $R_{x^{*} x}(\tau) \triangleq \mathrm{E}\left[x^{*}(t) x(t+\tau)\right]$ is the autocorrelation function (or complex coherence function) of the (stationary) source signal $x(t)$. In case of thermal light, which is an approximate model for spontaneously emitted radiation, for example in case of a light-emitting diode (LED), superluminescent diode (SLD), or erbium-doped fiber (EDF) source, $x(t)$ can be modeled as a circular complex Gaussian bandpass process [2], [3]. In case of an LED or SLD, the spectrum of the light is approximately Gaussian, such that we have

$$
R_{x^{*} x}(\tau)=2 P_{\text {in }} \exp \left(-\frac{\pi}{2}\left(\frac{\tau}{\tau_{c}}\right)^{2}\right) \exp \left(j 2 \pi f_{c} \tau\right)
$$

where $f_{c}$ is the center frequency and $\tau_{c}$ is the coherence time of the source, which is defined as

$$
\tau_{c} \equiv \frac{\int_{-\infty}^{\infty}\left|R_{x^{*} x}(\tau)\right|^{2} d \tau}{R_{x^{*} x}^{2}(0)}
$$

and could be described as the width of the coherence function, or as the time span over which one can reasonably predict the phase of the light wave. It can be verified that the relation between the coherence time $\tau_{c}$ and the spectral full-width at half-maximum (FWHM) bandwidth $\Delta f$ is given by

$$
\tau_{c}=\sqrt{\frac{2 \ln 2}{\pi}} \frac{1}{\Delta f} \approx \sqrt{\frac{2 \ln 2}{\pi}} \frac{\lambda_{c}^{2}}{c_{0}} \frac{1}{\Delta \lambda}
$$

where $\lambda_{c}$ is the center wavelength, $c_{0}$ is the speed of light in vacuum and $\Delta \lambda$ is the linewidth of the source.

The path imbalance $T_{\mathrm{Tx}}$ of the MZI is chosen to be much larger than this coherence time $\tau_{c}$, such that the two mutually shifted light waves do not interfere coherently. Hence, only the intensity modulation $P_{\text {mod }}(t)$ (and not the phase modulation $\left.\phi_{\text {mod }}(t)\right)$ results in a visible intensity modulation in $y(t)$ : the expected (ensemble average) transmitted power at time $t$ is

$$
\frac{1}{2} R_{y^{*} y}(t, t)=\frac{1}{2} P_{\text {in }} P_{\text {mod }}(t)
$$

which is independent of $\phi_{\bmod }(t)$.

The receiver consists of another two-arm MZI (having path imbalance $T_{\mathrm{Rx}}$ ), a balanced photodiode pair and a transimpedance amplifier (TIA) having impulse response $h(t)$. A balanced detector configuration is considered, as it is proven to result in a better noise performance than a single-ended detector. Moreover, balanced detection is required when intensity modulation is being used [4]. By neglecting the attenuation and chromatic dispersion in the fiber, the electrical fields at the upper and lower output ports of the MZI can be written as

$$
\begin{aligned}
& z_{1}(t)=\frac{1}{2}\left[y(t)-y\left(t-T_{\mathrm{Rx}}\right)\right] \\
& z_{2}(t)=\frac{1}{2} j\left[y(t)+y\left(t-T_{\mathrm{Rx}}\right)\right]
\end{aligned}
$$

where the propagation delay of the transmission fiber is ignored as it only introduces an extra delay in the output signal of the system. 
When the photodiodes are assumed to be perfectly linear with identical responsivities $R_{p d}$, the output voltage $V_{\text {out }}(t)$ of the balanced receiver has expected value

$$
\begin{aligned}
\mathrm{E}\left[V_{\text {out }}(t)\right]= & \frac{1}{2} R_{p d}\left(h \otimes \mathrm{E}\left[\left|z_{1}\right|^{2}-\left|z_{2}\right|^{2}\right]\right)(t) \\
= & -\frac{1}{2} R_{p d} \int h(t-\tau) \\
& \cdot \operatorname{Re}\left\{R_{y^{*} y}\left(\tau-T_{\mathrm{Rx}}, \tau\right)\right\} d \tau
\end{aligned}
$$

where $\otimes$ denotes convolution and $\operatorname{Re}\{$.$\} denotes real part. As a$ result, the receiver estimates the degree of correlation between $y(t)$ and its delayed version $y\left(t-T_{\mathrm{Rx}}\right)$. Using (3), and incorporating that both $T_{\mathrm{Tx}}$ and $T_{\mathrm{Rx}}$ are assumed to be much larger than the coherence time $\tau_{c}$, this simplifies to

$$
\begin{aligned}
\mathrm{E}\left[V_{\text {out }}(t)\right]= & \frac{1}{8} R_{p d} \operatorname{Re}\left\{R_{x^{*} x}\left(T_{\mathrm{Rx}}-T_{\mathrm{Tx}}\right)\right. \\
& \left.\cdot \int h(t-\tau) P_{\mathrm{mod}}(\tau) \exp \left(j \phi_{\mathrm{mod}}(\tau)\right) d \tau\right\} .
\end{aligned}
$$

As a result, the average output signal depends on the relation between $T_{\mathrm{Rx}}$ and $T_{\mathrm{Tx}}$.

- When the difference between $T_{\mathrm{Rx}}$ and $T_{\mathrm{Tx}}$ is much larger than $\tau_{c}$, all light waves entering the balanced receiver are mutually incoherent, resulting in an expected output current which is negligible.

- When the difference between $T_{\mathrm{Rx}}$ and $T_{\mathrm{Tx}}$ is much smaller than $\tau_{c}$, however, the light wave taking the lower path in the transmitter and the upper path in the receiver interferes coherently with the light wave taking the upper path in the transmitter and the lower path in the receiver. Using (4), the following expected output signal results:

$$
\begin{aligned}
& \mathrm{E}\left[V_{\mathrm{out}}(t)\right]=\frac{1}{4} R_{p d} P_{\text {in }} \int h(t-\tau) P_{\bmod }(\tau) \\
& \cdot \cos \left(2 \pi f_{c}\left(T_{\mathrm{Rx}}-T_{\mathrm{Tx}}\right)+\phi_{\bmod }(\tau)\right) d \tau .
\end{aligned}
$$

(For intermediate values of $\left|T_{\mathrm{Rx}}-T_{\mathrm{Tx}}\right|$, partially coherent interference occurs, which is of no interest in the context of CM systems.)

When binary PSK modulation is performed $\left(\phi_{\text {mod }}=0\right.$ for a binary zero, $\phi_{\text {mod }}=\pi$ for a binary one and $P_{\text {mod }}=1$ ), a (filtered) polar NRZ output signal appears at the output of the matched receiver. When OOK modulation is performed $\left(P_{\bmod }=0\right.$ for a binary zero, $P_{\bmod }=1$ for a binary one and $\phi_{\bmod }=0$ ), a (filtered) unipolar NRZ signal results. In both cases, the amplitude of the output signal is proportional to $\cos \left(2 \pi f_{c}\left(T_{\mathrm{Rx}}-T_{\mathrm{Tx}}\right)\right)$, which renders the output signal very sensitive for small changes in $\left|T_{\mathrm{Rx}}-T_{\mathrm{Tx}}\right|$ (since $f_{c}$ is very large). We will come back to this in Section III.

\section{B. Multiplexing by Means of a Parallel Array}

Although several other system topologies are known for coherence multiplexing [5], we will consider the parallel array [6], [7], because it is the only topology that enables intensity modulation by direct modulation, and it provides the most flexibility in locating the transmitters [8].

Consider the situation with $N$ transmitters and $N$ receivers that are mutually connected either by an $N \times N$-star coupler

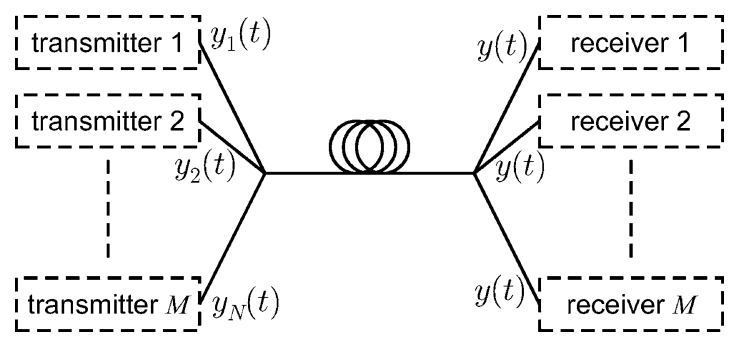

Fig. 2. A coherence multiplex system with $N$ transmitters and $N$ receivers in a parallel array configuration.

or an $N \times 1$-combiner, a common fiber and a $1 \times N$-splitter, as shown in Fig. 2. In that case, each transmitter has its own source signal $x_{i}(t)$, path imbalance $T_{\mathrm{Tx}, i}$ and modulating signals $P_{\text {mod, } i}(t)$ and $\phi_{\text {mod, }, i}(t)$. Each transmitted light wave $y_{i}(t)$ encounters a loss $L_{X}$ by traveling from transmitter $i$ to any receiver, including coupling losses, splitting losses, and propagation losses. Hence, when irrelevant group and phase delays in fibers and couplers are ignored, as well as group delay dispersion, then each receiver receives a signal

$$
\begin{aligned}
y(t)= & \frac{1}{\sqrt{L_{X}}} \sum_{i=1}^{N} y_{i}(t) \\
= & \frac{1}{2 \sqrt{L_{X}}} \sum_{i=1}^{N} \sqrt{P_{\bmod , i}(t)} \\
& \cdot\left[x_{i}(t)-x_{i}\left(t-T_{\mathrm{Tx}, i}\right) \exp \left(j \phi_{\bmod , i}(t)\right)\right] .
\end{aligned}
$$

Assuming that all the source signals are independent and have the same autocorrelation function $R_{x^{*} x}(\tau)$, the autocorrelation function of $y(t)$ can be written as

$$
\begin{aligned}
R_{y^{*} y}\left(t_{1}, t_{2}\right)= & \frac{1}{4 L_{X}} \sum_{i=1}^{N} \sqrt{P_{\mathrm{mod}, i}\left(t_{1}\right) P_{\mathrm{mod}, i}\left(t_{2}\right)} \\
\cdot & {\left[R_{x^{*} x}\left(t_{2}-t_{1}\right)\right.} \\
& -R_{x^{*} x}\left(t_{2}-t_{1}-T_{\mathrm{Tx}, i}\right) \exp \left(j \phi_{\bmod , i}\left(t_{2}\right)\right) \\
& -R_{x^{*} x}\left(t_{2}-t_{1}+T_{\mathrm{Tx}, i}\right) \exp \left(-j \phi_{\mathrm{mod}, i}\left(t_{1}\right)\right) \\
& +R_{x^{*} x}\left(t_{2}-t_{1}\right) \\
& \left.\cdot \exp \left(j\left(\phi_{\mathrm{mod}, i}\left(t_{2}\right)-\phi_{\mathrm{mod}, i}\left(t_{1}\right)\right)\right)\right] .
\end{aligned}
$$

When each receiver is matched to its corresponding transmitter (so $\left|T_{\mathrm{Rx}, r}-T_{\mathrm{Tx}, i}\right| \ll \tau_{c}$ when $i=r$ ) and the path delays in different transmitters are spaced apart much more than the coherence time (such that $\left|T_{\mathrm{Rx}, r}-T_{\mathrm{Tx}, i}\right| \gg \tau_{c}$ when $i \neq r$, for all $i$ and $r$ ), then, using (10) it can easily be verified that the output signal of a particular receiver $r$ only depends on the modulating signals of the corresponding transmitter $r$

$$
\begin{aligned}
& \mathrm{E}\left[V_{\mathrm{out}, r}(t)\right]=\frac{R_{p d} P_{\mathrm{in}}}{4 L_{X}} \int h(t-\tau) P_{\mathrm{mod}, r}(\tau) \\
& \cdot \cos \left(2 \pi f_{c}\left(T_{\mathrm{Rx}, r}-T_{\mathrm{Tx}, r}\right)+\phi_{\mathrm{mod}, r}(\tau)\right) d \tau .
\end{aligned}
$$

Obviously, small fluctuations in the path imbalances will not introduce interchannel interference, so the $\mathrm{CM}$ concept is very robust as far as crosstalk is concerned. Unfortunately, this does not hold for the stability of the output signal, as will be explained in the next section. 


\section{Phase SENSITIVITY IsSUE AND KNOWN SOLUTIONS}

When coherent interference occurs, the amplitude of the output signal is depending on the phase difference $\Delta \phi+\phi_{\text {mod }}(t)$, where $\Delta \phi=2 \pi f_{c}\left(T_{\mathrm{Rx}}-T_{\mathrm{Tx}}\right)$. Since the optical carrier frequency $f_{c}$ is very large, only small mismatches between $T_{\mathrm{Rx}}$ and $T_{\mathrm{Tx}}$ already result in a significant amplitude reduction. This can be caused by fabrication tolerances or aging of components. As a result, some kind of phase synchronization mechanism is required such that $f_{c}\left(T_{\mathrm{Rx}}-T_{\mathrm{Tx}}\right)$ is a (small) integer number, for example by applying a feedback loop and a frequency dithering technique [9].

The feedback loop can be omitted when self-heterodyning is employed instead of self-homodyning [10]. This can be done by inserting a frequency shifter-for example, an acoustooptic modulator (AOM) - in one of the arms of either the transmitter or receiver, resulting in an expected output signal

$$
\begin{aligned}
\mathrm{E}\left[V_{\text {out }, r}\right]=\frac{R_{p d} P_{\text {in }}}{4 L_{X}} \int h(t-\tau) P_{\bmod , r}(\tau) \\
\cdot \cos \left(2 \pi f_{s} \tau+\Delta \phi_{r}+\phi_{\bmod , r}(\tau)\right) d \tau
\end{aligned}
$$

where $f_{s}$ corresponds to the frequency shift. The disadvantage of this solution is that the output current becomes a bandpass signal instead of a baseband signal (so obviously, $h(t)$ should correspond to a bandpass response in this case), resulting in an inherent loss of $3 \mathrm{~dB}$ in signal-to-noise ratio (SNR). Since the modulation rate is restricted to a fraction of the intermediate frequency $f_{s}$, very high processing frequencies are required if high datarates are to be accommodated. Moreover, the AOM will introduce insertion loss, which even further reduces the SNR.

Both the self-homodyning and the self-heterodyning solutions are rather complicated, which conflicts with the main motivation for $\mathrm{CM}$ of keeping the total system costs low. Therefore, a new $\mathrm{CM}$ receiver structure is proposed in this paper, which does not require a feedback loop or a frequency shifter, as it is based on phase diversity detection.

\section{OUtPut Stabilization by MEAns of Phase Diversity}

Phase diversity is a detection technique that has extensively been applied in coherent optical communication systems [11], for compensating the phase noise between the received and locally generated optical carrier. Although the possibility of stabilizing a CM receiver's output signal by means of phase diversity had already been suggested in 1987 by Healey [12], no closer studies about the feasibility of such a receiver were published (to our knowledge) until our proposal in 2001 [13].

The idea of phase diversity is that the $2 \times 2$ coupler and the two photodiodes in the rightmost part of the receiver in Fig. 1 are replaced by a multiport coupler and a corresponding number of photodiodes. As a result of the diversity in the phase transfer of the multiport coupler, multiple interference products are obtained that are similar to (15), but each with a different phase-offset in the cosine. Proper combining of these interference products then provides an output signal that does not depend on the phase difference between the coherently mixed light waves. Hence, neither a phase synchronization mechanism nor a frequency shifter is required in order to stabilize the output signal of such a receiver.

In the following sections, two particular phase diversity networks will be described, one being based on a $4 \times 4$ optical hybrid and the other being based on a $3 \times 3$ optical hybrid. As possible modulation schemes, on-off keying (OOK), binary differential phase shift keying (binary DPSK) and $M$-ary differential phase shift keying ( $M$-ary DPSK) will be considered.

\section{A. Four-Way Phase Diversity Detection of $O O K$}

The most straightforward solution for performing phase diversity detection is by using a $4 \times 4$ phase diversity coupler [14], for example a multimode interference (MMI) coupler. The corresponding detection scheme is shown in Fig. 3. The coupler is assumed to have an ideal transfer matrix

$$
\left[H_{4}\right]=\frac{1}{2}\left[\begin{array}{cccc}
\exp \left(j \frac{\pi}{4}\right) & -1 & 1 & \exp \left(j \frac{\pi}{4}\right) \\
-1 & \exp \left(j \frac{\pi}{4}\right) & \exp \left(j \frac{\pi}{4}\right) & 1 \\
1 & \exp \left(j \frac{\pi}{4}\right) & \exp \left(j \frac{\pi}{4}\right) & -1 \\
\exp \left(j \frac{\pi}{4}\right) & 1 & -1 & \exp \left(j \frac{\pi}{4}\right)
\end{array}\right] .
$$

By Heaton and Jenkins' definition [15], this corresponds to a 7 $\times 7$-MMI of which the odd-numbered inputs and outputs are used. When applied in a multitransmitter system as described in Section II-B, the electrical fields at the output of the MMI of a particular receiver $r$ can be written as

$$
\begin{aligned}
& z_{r, 1}(t)=\frac{1}{2 \sqrt{2}}\left[y(t) \exp \left(j \frac{\pi}{4}\right)+j y\left(t-T_{\mathrm{Rx}, r}\right)\right] \\
& z_{r, 2}(t)=\frac{1}{2 \sqrt{2}}\left[-y(t)+y\left(t-T_{\mathrm{Rx}, r}\right) \exp \left(j \frac{3 \pi}{4}\right)\right] \\
& z_{r, 3}(t)=\frac{1}{2 \sqrt{2}}\left[y(t)+y\left(t-T_{\mathrm{Rx}, r}\right) \exp \left(j \frac{3 \pi}{4}\right)\right] \\
& z_{r, 4}(t)=\frac{1}{2 \sqrt{2}}\left[y(t) \exp \left(j \frac{\pi}{4}\right)-j y\left(t-T_{\mathrm{Rx}, r}\right)\right] .
\end{aligned}
$$

Using (14), the output voltages of the amplifiers have expected values that are found to be

$$
\begin{aligned}
& \mathrm{E}\left[V_{I, r}(t)\right]=\frac{1}{2} R_{p d}\left(h \otimes \mathrm{E}\left[\left|z_{r, 2}\right|^{2}-\left|z_{r, 3}\right|^{2}\right]\right)(t) \\
& =\frac{1}{4} R_{p d} \int h(t-\tau) \\
& \text { - } \operatorname{Re}\left\{R_{y^{*} y}\left(\tau-T_{\mathrm{Rx}, r}, \tau\right) \exp \left(j \frac{\pi}{4}\right)\right\} d \tau \\
& =\frac{R_{p d} P_{\text {in }}}{8 L_{X}} \int h(t-\tau) P_{\bmod , r}(\tau) \\
& \cdot \cos \left(\Delta \phi_{r}+\phi_{\bmod , r}(\tau)-\frac{3 \pi}{4}\right) d \tau \\
& \mathrm{E}\left[V_{Q, r}(t)\right]=\frac{1}{2} R_{p d}\left(h \otimes \mathrm{E}\left[\left|z_{r, 1}\right|^{2}-\left|z_{r, 4}\right|^{2}\right]\right)(t) \\
& =\frac{1}{4} R_{p d} \int h(t-\tau) \\
& \cdot \operatorname{Re}\left\{R_{y^{*} y}\left(\tau-T_{\mathrm{Rx}}, \tau\right) \exp \left(-j \frac{\pi}{4}\right)\right\} d \tau \\
& =\frac{R_{p d} P_{\text {in }}}{8 L_{X}} \int h(t-\tau) P_{\bmod , r}(\tau) \\
& \cdot \cos \left(\Delta \phi_{r}+\phi_{\bmod , r}(\tau)+\frac{3 \pi}{4}\right) d \tau \text {. }
\end{aligned}
$$




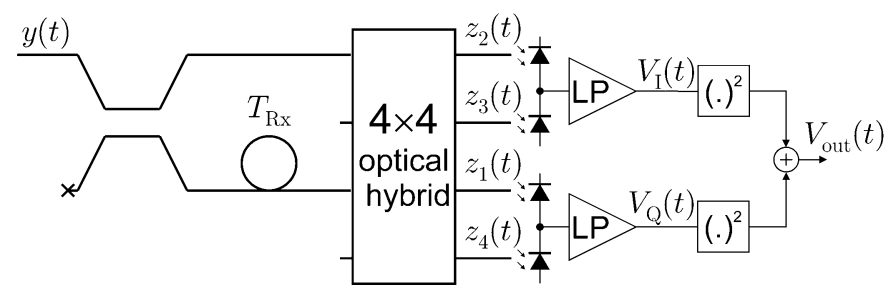

Fig. 3. Four-way OOK phase diversity receiver. Note that the order of the output ports of the $4 \times 4$ optical hybrid has been interchanged for reasons of graphical presentation.

Now assume that digital modulation is performed, with a rectangular symbol shape of length $T_{s}$, so we can write

$$
\begin{aligned}
& P_{\mathrm{mod}, r}(t)=\sum_{l} P_{\mathrm{mod}, r}\left(l \cdot T_{s}\right) \Pi\left(\frac{t-l \cdot T_{s}}{T_{s}}\right) \\
& \phi_{\mathrm{mod}, r}(t)=\sum_{l} \phi_{\mathrm{mod}, r}\left(l \cdot T_{s}\right) \Pi\left(\frac{t-l \cdot T_{s}}{T_{s}}\right)
\end{aligned}
$$

where

$$
\Pi(x) \triangleq \begin{cases}1, & \text { if }|x|<\frac{1}{2} \\ 0, & \text { if }|x|>\frac{1}{2}\end{cases}
$$

To optimize the SNR prior to detection, $h(t)$ is assumed to be matched to these information symbols, so we have

$$
h(t)=G \cdot \Pi\left(\frac{t}{T_{s}}\right)
$$

where $G$ is the gain of the TIA. As a result, we can write at the optimum sampling instants $l \cdot T_{s}$

$$
\begin{aligned}
\mathrm{E}\left[V_{I, r}\left(l \cdot T_{s}\right)\right]= & \frac{R_{p d} P_{\mathrm{in}} G \cdot T_{s}}{8 L_{X}} P_{\mathrm{mod}, r}\left(l \cdot T_{s}\right) \\
& \cdot \cos \left(\Delta \phi_{r}+\phi_{\mathrm{mod}, r}\left(l \cdot T_{s}\right)-\frac{3 \pi}{4}\right) \\
\mathrm{E}\left[V_{Q, r}\left(l \cdot T_{s}\right)\right]= & \frac{R_{p d} P_{\mathrm{in}} G \cdot T_{s}}{8 L_{X}} P_{\mathrm{mod}, r}\left(l \cdot T_{s}\right) \\
& \cdot \cos \left(\Delta \phi_{r}+\phi_{\mathrm{mod}, r}\left(l \cdot T_{s}\right)+\frac{3 \pi}{4}\right) .
\end{aligned}
$$

When the SNR after amplification and matched filtering is very high, the expected value of the output signal at the sampling instants can be approximated as

$$
\begin{aligned}
\mathrm{E}\left[V_{\text {out }}\left(l \cdot T_{s}\right)\right] & =\mathrm{E}\left[V_{I}^{2}\left(l \cdot T_{s}\right)+V_{Q}^{2}\left(l \cdot T_{s}\right)\right] \\
& \approx \mathrm{E}^{2}\left[V_{I}\left(l \cdot T_{s}\right)\right]+\mathrm{E}^{2}\left[V_{Q}\left(l \cdot T_{s}\right)\right] \\
& =\frac{R_{p d}^{2} P_{\text {in }}^{2} G^{2} T_{s}^{2}}{64 L_{X}^{2}} P_{\bmod , r}^{2}\left(l \cdot T_{s}\right) .
\end{aligned}
$$

Obviously, since $\Delta \phi_{r}$ does not appear in this expression, the output signal at the optimum sampling instants no longer depends on the difference between $T_{\mathrm{Rx}, r}$ and $T_{\mathrm{Tx}, r}$, as long as it is much smaller than the coherence time. Note, however, that the output signal at the sampling instants is also independent of $\phi_{\text {mod, } r}$. Hence, this specific type of receiver is solely suitable for intensity modulation, like on-off keying (OOK). In a later subsection, a specific receiver for demodulating phase modulated
CM signals will be discussed, but first it will be shown how the same detection operation can be performed using a $3 \times 3$-coupler.

\section{B. Three-Way Phase Diversity Detection of OOK}

In Fig. 4 a CM receiver based on three-way balanced optical phase diversity is shown. The transfer function of the $3 \times 3$-coupler is given by [16]

$$
\left[H_{3}\right]=\frac{1}{\sqrt{3}}\left[\begin{array}{ccc}
1 & 1 & 1 \\
1 & \exp \left(j \frac{2 \pi}{3}\right) & \exp \left(-j \frac{2 \pi}{3}\right) \\
1 & \exp \left(-j \frac{2 \pi}{3}\right) & \exp \left(j \frac{2 \pi}{3}\right)
\end{array}\right] .
$$

The calculation of the expected output signals of the differential amplifiers at the optimum sampling instants is similar to the calculation in the previous subsection, and will result in

$$
\begin{aligned}
\mathrm{E}\left[V_{\mathrm{a}, r}\left(l \cdot T_{s}\right)\right]= & \frac{R_{p d} P_{\mathrm{in}} G \cdot T_{s}}{4 \sqrt{3} L_{X}} P_{\mathrm{mod}, r}\left(l \cdot T_{s}\right) \\
& \cdot \cos \left(\Delta \phi_{r}+\phi_{\mathrm{mod}, r}\left(l \cdot T_{s}\right)+\frac{2 \pi}{3}\right) \\
\mathrm{E}\left[V_{\mathrm{b}, r}\left(l \cdot T_{s}\right)\right]= & \frac{R_{p d} P_{\mathrm{in}} G \cdot T_{s}}{4 \sqrt{3} L_{X}} P_{\mathrm{mod}, r}\left(l \cdot T_{s}\right) \\
& \cdot \cos \left(\Delta \phi_{r}+\phi_{\mathrm{mod}, r}\left(l \cdot T_{s}\right)\right) \\
\mathrm{E}\left[V_{c, r}\left(l \cdot T_{s}\right)\right]= & \frac{R_{p d} P_{\mathrm{in}} G \cdot T_{s}}{4 \sqrt{3} L_{X}} P_{\mathrm{mod}, r}\left(l \cdot T_{s}\right) \\
& \cdot \cos \left(\Delta \phi_{r}+\phi_{\mathrm{mod}, r}\left(l \cdot T_{s}\right)-\frac{2 \pi}{3}\right) .
\end{aligned}
$$

Consequently, the expected value of the output signal at the optimum sampling instants can be approximated by

$$
\begin{aligned}
\mathrm{E}\left[V_{\text {out }}\left(l \cdot T_{s}\right)\right]= & \mathrm{E}\left[V_{\mathrm{a}}^{2}\left(l \cdot T_{s}\right)+V_{\mathrm{b}}^{2}\left(l \cdot T_{s}\right)+V_{c}^{2}\left(l \cdot T_{s}\right)\right] \\
\approx & \mathrm{E}^{2}\left[V_{\mathrm{a}}\left(l \cdot T_{s}\right)\right]+\mathrm{E}^{2}\left[V_{\mathrm{b}}\left(l \cdot T_{s}\right)\right] \\
& +\mathrm{E}^{2}\left[V_{c}\left(l \cdot T_{s}\right)\right] \\
= & \frac{R_{p d}^{2} P_{\mathrm{in}}^{2} G^{2} T_{s}^{2}}{32 L_{X}^{2}} P_{\mathrm{mod}, r}^{2}\left(l \cdot T_{s}\right)
\end{aligned}
$$

Obviously, (35) is similar to (30). The advantage of the three-way phase diversity receiver over the four-way phase diversity receiver, however, is that the $3 \times 3$-coupler can be fabricated very easily, simply by fusing three fibers. The $4 \times$ 4-coupler has to be fabricated either as an integrated optical device or as an all-fiber hybrid that requires very strict tuning. Therefore, the three-way receiver is more suitable for experimental prototyping purposes. The disadvantage of a fiber setup, however, is that a polarization controller is required for matching the polarization of the coherently mixed light waves, which is not desirable in a final product. Therefore, the advantage of the four-way receiver is that the entire optical interferometer (including the $4 \times 4-\mathrm{MMI}$ ) can be realized as an optical integrated circuit, hence maintaining polarization states. Another advantage of the four-way phase diversity receiver is that the balanced currents can simply be obtained by two distinct balanced receivers, whereas in the three-way solution, these have to be obtained either by a complicated circular balancing circuit, or by three differential amplifiers with a high 


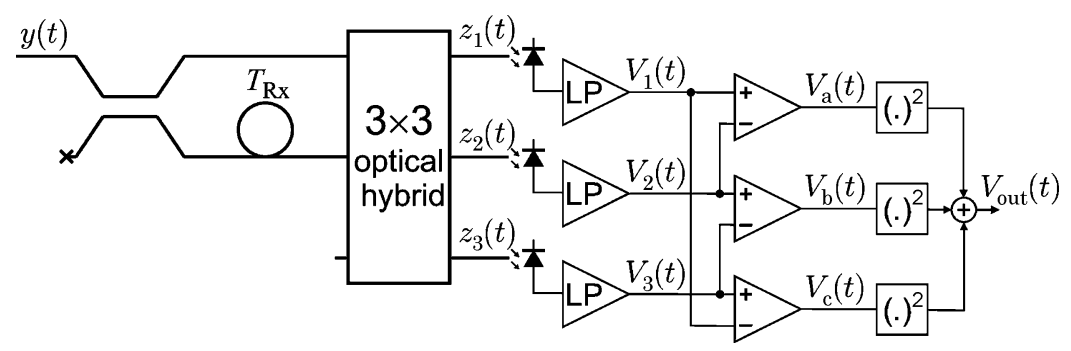

Fig. 4. Three-way OOK phase diversity receiver.

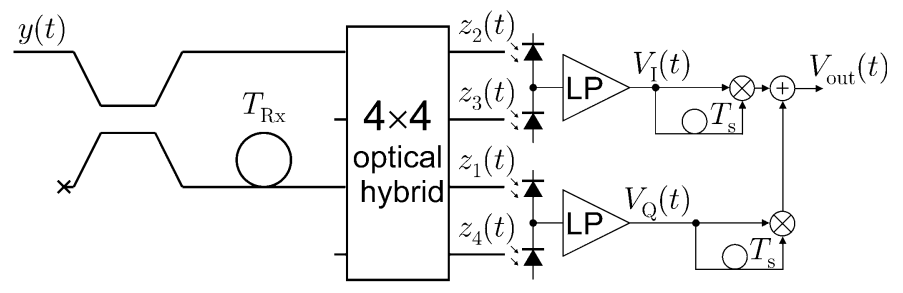

Fig. 5. Four-way binary DPSK phase diversity receiver.

common-mode rejection ratio (CMRR). The performances of the three- and four-way receiver will be compared in Section V.

\section{Phase Diversity Detection of Binary DPSK}

Although phase diversity is not suitable for detecting the absolute phase difference between the coherently mixed light waves, it can be used to detect changes in this phase difference; digital transmission can be accomplished by performing differential phase shift keying (DPSK) modulation, and by replacing the squarers in the receiver by a delay-and-multiply circuit: see Fig. 5 [17]. The delay $T_{s}$ in the delay-and-multiply circuit corresponds to one symbol period. In that case, we have $P_{\text {mod, } r}(t)=1$, and successive values of $\phi_{\bmod , r}\left(l \cdot T_{s}\right)$ are the same for a binary 1 and have a difference of $\pi$ for a binary zero. The resulting output signal can then be shown to have expected value

$$
\begin{aligned}
\mathrm{E}\left[V_{\text {out }, r}\left(l \cdot T_{s}\right)\right]= & \mathrm{E}\left[V_{I, r}\left(l \cdot T_{s}\right) V_{I, r}\left((l-1) T_{s}\right)\right. \\
& \left.+V_{Q, r}\left(l \cdot T_{s}\right) V_{Q, r}\left((l-1) T_{s}\right)\right] \\
= & \mathrm{E}\left[V_{I, r}\left(l \cdot T_{s}\right)\right] \mathrm{E}\left[V_{I, r}\left((l-1) T_{s}\right)\right] \\
& +\mathrm{E}\left[V_{Q, r}\left(l \cdot T_{s}\right)\right] \mathrm{E}\left[V_{Q, r}\left((l-1) T_{s}\right)\right] \\
= & \frac{R_{p d}^{2} P_{\mathrm{in}}^{2} G^{2} T_{s}^{2}}{64 L_{X}^{2}} \\
& \cdot \cos \left(\phi_{\bmod , r}\left(l \cdot T_{s}\right)\right. \\
& \left.\quad-\phi_{\bmod , r}\left((l-1) T_{s}\right)\right)
\end{aligned}
$$

which is positive for a binary one, and negative for a binary zero, irrespective of small fluctuations in the optical path imbalances in transmitter or receiver. Moreover, note that the delays $T_{s}$ do not require strict matching either, since the electrical signals $V_{I}(t)$ and $V_{Q}(t)$ are baseband signals.

One can verify that a similar operation can be performed by means of a three-way phase diversity receiver, when the three squarers in Fig. 4 are replaced by delay-and-multiply circuits.

The advantages of using DPSK instead of OOK modulation is that the signal portions that interfering channels contribute to the

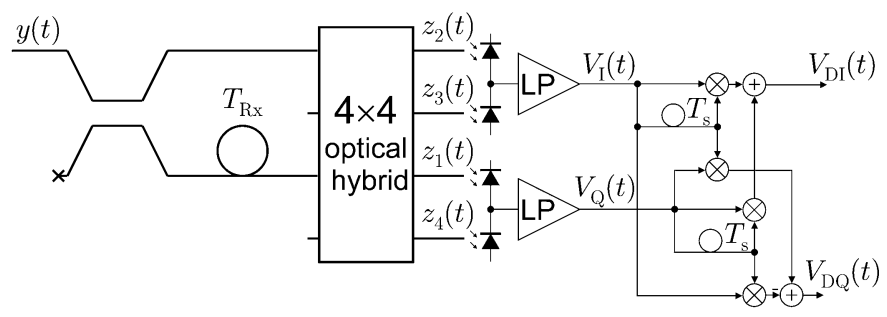

Fig. 6. Four-way $M$-ary DPSK phase diversity receiver.

photodiode currents appear as dc terms, whereas the (desired) interference products are antipodal. Therefore, balancing can now be performed ac-coupled, hence, putting less requirement on the CMRR of the balancing circuit.

\section{Phase Diversity Detection of $M$-ary DPSK}

The binary DPSK scheme can be extended to an $M$-ary DPSK receiver [18] as shown in Fig. 6. Obviously, $V_{\mathrm{DI}, r}(t)$ is the same as the output signal of the binary DPSK receiver, and for $V_{\mathrm{DQ}, r}(t)$, one can find

$$
\begin{aligned}
\mathrm{E}\left[V_{\mathrm{DQ}, r}\left(l \cdot T_{s}\right)\right]=\frac{R_{p d}^{2} P_{\mathrm{in}}^{2} G^{2} T_{s}^{2}}{64 L_{X}^{2}} \\
\cdot \sin \left(\phi_{\bmod , r}\left(l \cdot T_{s}\right)-\phi_{\mathrm{mod}, r}\left((l-1) T_{s}\right)\right) .
\end{aligned}
$$

As a result, the transmitted symbols can be extracted by conventional PSK decoding [19]. For DQPSK for example, the bits can be extracted by directly thresholding $V_{\mathrm{DI}, r}$ and $V_{\mathrm{DQ}, r}$, provided that the phase increments in the transmitter are chosen as odd multiples of $\pi / 4$ ( $\pi / 4$-DQPSK).

The advantage of using $M$-ary DPSK rather than binary DPSK is that the symbol time increases with the number of possible symbol levels $M$. Hence, both the modulation speed in the transmitter and the signal processing speed in the receiver can be reduced, and moreover, the received signal will be less susceptible for intersymbol interference caused by fiber dispersion [20].

$M$-ary DPSK can also be demodulated using a three-way phase diversity receiver. It can be shown that this can for example be done by constructing signals $V_{I, r}(t)$ and $V_{Q, r}(t)$ from the TIA output signals $V_{r, 1}(t), V_{r, 2}(t)$, and $V_{r, 3}(t)$ as

$$
\begin{aligned}
V_{I, r}(t) & =V_{r, 1}(t)-V_{r, 2}(t) \\
V_{Q, r}(t) & =\frac{1}{\sqrt{3}}\left(V_{r, 1}(t)+V_{r, 2}(t)-2 V_{r, 3}(t)\right) .
\end{aligned}
$$

The remaining operations are the same as in the four-way $M$-ary DPSK phase diversity receiver. 


\section{PERformance COMPARISON OF VARIOUS RECEIVER STRUCTURES}

The performance of the CM receivers is mainly limited by three types of noise [3]:

- Source-induced noise, which results from the random behavior of the source signal, causing intensity fluctuations in the detected light waves. It can be categorized into intensity-induced noise and phase-induced noise [3]. Intensity-induced noise affects the coherent mixing product and results from intensity fluctuations in the light wave emitted by the source. Phase-induced noise [or optical beat interference (OBI) noise, or interferometric noise], results from incoherent mixing of light waves.

- Shot noise, which results from the random arrival times of photons.

- Thermal receiver noise, which results from the random motion of charge carriers in the resistors and transistors of the receiver electronics.

The performance of the various receiver structures can be evaluated in terms of their bit error rate (BER), which will be derived using the following procedure:

- Expressions will be derived that relate the expected values, variances and covariances of the balanced signals at the optimum sampling instant to the autocorrelation function of the received optical signal that was derived in (14);

- By means of a Gaussian approximation, the conditional probability density functions of the decision sample will be found;

- Using the conditional probability density functions, the value of the optimum decision threshold can be found, and subsequently, the BER can be calculated.

First, a description of the system model will be given.

\section{A. General Assumptions}

For simplicity, it is assumed that the light waves entering the receiver all have matched polarization states, hence providing an upper bound to the noise power due to OBI noise. Moreover, it is assumed that each receiver receives an equal amount of power from each transmitter.

The photon arrival process is modeled as an imhomogeneous doubly stochastic Poisson process [2]. The photocurrents are amplified by a transimpedance amplifier (TIA) with impulse response $h(t)$. It is assumed that thermal receiver noise is dominated by the noise that is generated by the TIA. The equivalent input noise current $i_{\mathrm{th}}(t)$ of this noise is assumed to have a flat power spectral density function $S_{i_{\mathrm{th}}}(f)=S_{\mathrm{th}}$. Hence, when the (random) power of a light wave that is detected by a photodiode is denoted by $P(t)$, then the output voltage $V_{\text {out }}(t)$ of the TIA has expected value

and variance

$$
\mathrm{E}\left[V_{\text {out }}(t)\right]=R_{p d} \int h(t-\tau) \mathrm{E}[P(\tau)] d \tau
$$

$$
\begin{gathered}
\sigma_{V_{\text {out }}(t)}^{2}=R_{p d}^{2} \iint h\left(t-\tau_{1}\right) h\left(t-\tau_{2}\right) C_{P P}\left(\tau_{1}, \tau_{2}\right) d \tau_{1} d \tau_{2} \\
+R_{p d} e \int h^{2}(t-\tau) \mathrm{E}[P(\tau)] d \tau+S_{\text {th }} \int h^{2}(\tau) d \tau
\end{gathered}
$$

where $C_{P P}\left(t_{1}, t_{2}\right)$ denotes the covariance function of $P(t)$ and $e$ corresponds to the charge of an electron. (The three lines in this equation actually correspond to source-induced noise, shot noise and thermal noise, respectively.)

It can be proven that the output voltages $V_{1}(t)$ and $V_{2}(t)$ of two identical TIA's amplifying photocurrents corresponding to light waves with (random) powers $P_{1}(t)$ and $P_{2}(t)$, respectively, have covariance at time $t$ that is given by

$C_{V_{1} V_{2}}(t, t)=R_{p d}^{2} \iint h\left(t-\tau_{1}\right) h\left(t-\tau_{2}\right) C_{P_{1} P_{2}}\left(\tau_{1}, \tau_{2}\right) d \tau_{1} d \tau_{2}$

where $C_{P_{1} P_{2}}\left(t_{1}, t_{2}\right)$ denotes the cross covariance function of $P_{1}(t)$ and $P_{2}(t)$. It can be proven that the latter three formulas hold even for nonstationary light wave signals.

In case of OOK modulation, the instantaneous noise power in the receiver depends on the number of transmitters that are transmitting a binary one at that particular time instant. For simplicity, it is assumed that all transmitters are bit-synchronized, and that all transmitters transmit independent memoryless bit sequences in which binary zeroes and ones occur with identical probability $1 / 2$. Hence, when the number of interfering transmitters that are transmitting a binary one at time instant $l \cdot T_{s}$ is written as

$$
N_{1, l} \equiv \sum_{\substack{i=1 \\ i \neq r}}^{N} P_{\bmod , i}\left(l \cdot T_{s}\right)
$$

then $N_{1, l}$ can be modeled as a binomially distributed random variable

$$
P\left[N_{1, l}=n\right]=\left(\begin{array}{c}
N-1 \\
n
\end{array}\right)\left(\frac{1}{2}\right)^{N-1} .
$$

Hence, the total bit error probability for OOK modulation can be found by calculating the conditional bit error probabilities for given values of $N_{1, l}$ and $P_{\bmod , r}\left(l \cdot T_{s}\right)$, and summing the conditional bit error probabilities over all possible values of $N_{1, l}$ and $P_{\text {mod,r }}$, weighted by their individual probability distributions

$$
\begin{aligned}
& P_{e, \mathrm{OOK}}=\left(\frac{1}{2}\right)^{N} \sum_{n=0}^{N-1}\left(\begin{array}{c}
N-1 \\
n
\end{array}\right) \\
& \cdot\left[P_{e \mid N_{1, l}=n, P_{\mathrm{mod}, r}\left(l \cdot T_{s}\right)=0}+P_{e \mid N_{1, l}=n, P_{\mathrm{mod}, r}\left(l \cdot T_{s}\right)=1}\right] .
\end{aligned}
$$

The conditional error probabilities depend on the conditional probability density functions of the output sample of the receiver. This will be derived in the following sections. In the derivation of this conditional probability distribution, $N_{1, l}$ and $P_{\text {mod, } r}$ will-for the time being-be considered as deterministic variables, to simplify the notations of the various expected values and autocorrelation functions that are involved in the derivation.

\section{B. Four-Way Receivers}

Consider a particular four-way receiver $r$, which is matched to the corresponding transmitter $r$. If digital modulation and matched filtering is applied, the output signals $V_{I, r}(t)$ and $V_{Q, r}(t)$ have expected values at the optimum sampling instants 
$l \cdot T_{s}$ that are given by (28) and (29), respectively. Their variances and covariance can be found using (41) and (42). For the variance of $V_{I, r}\left(l \cdot T_{s}\right)$, this results in

$$
\begin{aligned}
& \sigma_{V_{I, r}\left(l \cdot T_{s}\right)}^{2}=\frac{1}{4} R_{p d}^{2} \iint\left[C_{\left|z_{r, 2}\right|^{2}\left|z_{r, 2}\right|^{2}}\left(\tau_{1}, \tau_{2}\right)\right. \\
& +C_{\left|z_{r, 3}\right|^{2}\left|z_{r, 3}\right|^{2}}\left(\tau_{1}, \tau_{2}\right) \\
& \left.-2 C_{\left|z_{r, 2}\right|^{2}\left|z_{r, 3}\right|^{2}}\left(\tau_{1}, \tau_{2}\right)\right] \\
& \text { - } h\left(l \cdot T_{s}-\tau_{1}\right) h\left(l \cdot T_{s}-\tau_{2}\right) d \tau_{1} d \tau_{2} \\
& +\frac{1}{2} R_{p d} e \int \mathrm{E}\left[\left|z_{r, 2}(\tau)\right|^{2}+\left|z_{r, 3}(\tau)\right|^{2}\right] \\
& \text { - } h^{2}\left(l \cdot T_{s}-\tau\right) d \tau+S_{\text {th }} \int h^{2}(\tau) d \tau \text {. }
\end{aligned}
$$

Separately calculating each of the covariance functions in, for example, the first integral is a laborious task. This can be avoided, however, if we use (18) through (21) and then apply the complex Gaussian moment theorem [2], which states that the joint moment of four circular complex Gaussian variables $X_{1}, X_{2}, X_{3}$, and $X_{4}$ is given by

$\mathrm{E}\left[X_{1} X_{2}^{*} X_{3} X_{4}^{*}\right]=\mathrm{E}\left[X_{1} X_{2}^{*}\right] \mathrm{E}\left[X_{3} X_{4}^{*}\right]+\mathrm{E}\left[X_{1} X_{4}^{*}\right] \mathrm{E}\left[X_{3} X_{2}^{*}\right]$.

Then we can write

$$
\begin{aligned}
& C_{\left|z_{r, 2}\right|^{2}\left|z_{r, 2}\right|^{2}}\left(\tau_{1}, \tau_{2}\right)+C_{\left|z_{r, 3}\right|^{2}\left|z_{r, 3}\right|^{2}}\left(\tau_{1}, \tau_{2}\right) \\
& -2 C_{\left|z_{r, 2}\right|^{2}\left|z_{r, 3}\right|^{2}}\left(\tau_{1}, \tau_{2}\right) \\
& \quad=\frac{1}{8} \operatorname{Re}\left\{R_{y^{*} y}\left(\tau_{1}, \tau_{2}\right) R_{y^{*} y}\left(\tau_{2}-T_{\mathrm{Rx}, r}, \tau_{1}-T_{\mathrm{Rx}, r}\right)\right. \\
& \left.\quad+j R_{y^{*} y}\left(\tau_{1}-T_{\mathrm{Rx}}, \tau_{2}\right) R_{y^{*} y}\left(\tau_{2}-T_{\mathrm{Rx}}, \tau_{1}\right)\right\} .
\end{aligned}
$$

Equation (48) can be evaluated by expanding the two products inside the braces. This can be done using (14), and can be shown to result into

$$
\begin{aligned}
& R_{y^{*} y}\left(\tau_{1}, \tau_{2}\right) R_{y^{*} y}\left(\tau_{2}-T_{\mathrm{Rx}, r}, \tau_{1}-T_{\mathrm{Rx}, r}\right) \\
& \approx \frac{1}{4 L_{X}^{2}} \sum_{i_{1}=1}^{N} \sum_{i_{2}=1}^{N}\left|R_{x^{*} x}\left(\tau_{2}-\tau_{1}\right)\right|^{2} \\
& \cdot \sqrt{P_{\bmod , i_{1}}\left(\tau_{1}\right) P_{\bmod , i_{1}}\left(\tau_{2}\right) P_{\bmod , i_{2}}\left(\tau_{1}\right) P_{\bmod , i_{2}}\left(\tau_{2}\right)} \\
&+\frac{1}{16 L_{X}^{2}} \sum_{i=1}^{N}\left[\left|R_{x^{*} x}\left(\tau_{2}-\tau_{1}-T_{\mathrm{Tx}, i}\right)\right|^{2}\right. \\
&\left.\quad+\left|R_{x^{*} x}\left(\tau_{2}-\tau_{1}+T_{\mathrm{Tx}, i}\right)\right|^{2}\right] \\
& R_{y^{*} y}\left(\tau_{1}-T_{\mathrm{Rx}}, \tau_{2}\right) R_{y^{*} y}\left(\tau_{2}-T_{\mathrm{Rx}}, \tau_{1}\right) \\
& \approx \frac{1}{16 L_{X}^{2}}\left|R_{x^{*} x}\left(\tau_{2}-\tau_{1}\right)\right|^{2} P_{\bmod , i}\left(\tau_{1}\right) P_{\bmod , i}\left(\tau_{2}\right) \\
& \cdot \exp \left(j\left(2 \Delta \phi_{r}+\phi_{\bmod , r}\left(\tau_{1}\right)+\phi_{\bmod , r}\left(\tau_{2}\right)\right)\right)
\end{aligned}
$$

In order to evaluate the second line of (46), we use (14), (19) and (20) to write

$$
\begin{aligned}
\mathrm{E}\left[\left|z_{r, 2}(\tau)\right|^{2}+\left|z_{r, 3}(\tau)\right|^{2}\right] & =\frac{1}{4} \mathrm{E}\left[|y(\tau)|^{2}+\left|y\left(\tau-T_{\mathrm{Rx}, r}\right)\right|^{2}\right] \\
& \approx \frac{P_{\text {in }}}{2 L_{X}} \sum_{i=1}^{N} P_{\bmod , i}(\tau) .
\end{aligned}
$$

Assuming that the integration time $T_{s}$ of the matched filter is much longer than the optical path delays and the coherence time $\tau_{c},(5),(48),(49),(50)$ and (51) can be used to write (46) as

$$
\begin{aligned}
& \sigma_{V_{I, r}\left(l \cdot T_{s}\right)}^{2}=\frac{R_{p d}^{2} P_{\mathrm{in}}^{2} G^{2} T_{s} \tau_{c}}{128 L_{X}^{2}} \\
& \cdot\left[4 \sum_{i_{1}=1}^{N} \sum_{i_{2}=1}^{N} P_{\bmod , i_{1}}\left(l \cdot T_{s}\right) P_{\bmod , i_{2}}\left(l \cdot T_{s}\right)\right. \\
& +2 \sum_{i=1}^{N} P_{\bmod , i}^{2}\left(l \cdot T_{s}\right)+P_{\bmod , r}^{2}\left(l \cdot T_{s}\right) \\
& \left.\cdot \cos \left(2 \Delta \phi_{r}+2 \phi_{\bmod , r}\left(l \cdot T_{s}\right)+\frac{\pi}{2}\right)\right] \\
& +\frac{R_{p d} P_{\mathrm{in}} e \cdot G^{2} T_{s}}{4 L_{X}} \sum_{i=1}^{N} P_{\bmod , i}\left(l \cdot T_{s}\right) \\
& +S_{\mathrm{th}} G^{2} T_{s} \text {. }
\end{aligned}
$$

Similar procedures can be used to find

$$
\begin{aligned}
& \sigma_{V_{Q, r}\left(l \cdot T_{s}\right)}^{2} \\
& =\frac{R_{p d}^{2} P_{\mathrm{in}}^{2} G^{2} T_{s} \tau_{c}}{128 L_{X}^{2}} \\
& \cdot\left[4 \sum_{i_{1}=1}^{N} \sum_{i_{2}=1}^{N} P_{\bmod , i_{1}}\left(l \cdot T_{s}\right) P_{\bmod , i_{2}}\left(l \cdot T_{s}\right)\right. \\
& +2 \sum_{i=1}^{N} P_{\bmod , i}^{2}\left(l \cdot T_{s}\right)+P_{\bmod , r}^{2}\left(l \cdot T_{s}\right) \\
& \left.\cdot \cos \left(2 \Delta \phi_{r}+2 \phi_{\bmod , r}\left(l \cdot T_{s}\right)-\frac{\pi}{2}\right)\right] \\
& +\frac{R_{p d} P_{\mathrm{in}} e \cdot G^{2} T_{s}}{4 L_{X}} \sum_{i=1}^{N} P_{\bmod , i}\left(l \cdot T_{s}\right) \\
& +S_{\mathrm{th}} G^{2} T_{s} \\
& C_{V_{I, r} V_{Q, r}}\left(l \cdot T_{s}, l \cdot T_{s}\right) \\
& =\frac{R_{p d}^{2} P_{\mathrm{in}}^{2} G^{2} T_{s} \tau_{c}}{128 L_{X}^{2}} P_{\bmod , r}^{2}\left(l \cdot T_{s}\right) \\
& \cdot \cos \left(2 \Delta \phi_{r}+2 \phi_{\bmod , r}\left(l \cdot T_{s}\right)\right) \text {. }
\end{aligned}
$$

When the output samples $V_{I, r}\left(l \cdot T_{s}\right)$ and $V_{Q, r}\left(l \cdot T_{s}\right)$ are written as a vector $\mathbf{V}_{r}\left(l \cdot T_{s}\right)=\left[V_{I, r}\left(l \cdot T_{s}\right), V_{Q, r}\left(l \cdot T_{s}\right)\right]^{\mathrm{T}}$, it has expected value

$$
\begin{aligned}
\mathrm{E}\left[\mathbf{V}_{r}\left(l \cdot T_{s}\right)\right]= & \frac{R_{p d} P_{\mathrm{in}} G \cdot T_{s}}{8 L_{X}} P_{\mathrm{mod}, r}\left(l \cdot T_{s}\right) \\
& \cdot\left[\begin{array}{l}
\cos \left(\Delta \phi_{r}+\phi_{\bmod , r}\left(l \cdot T_{s}\right)-\frac{3 \pi}{4}\right) \\
\cos \left(\Delta \phi_{r}+\phi_{\bmod , r}\left(l \cdot T_{s}\right)+\frac{3 \pi}{4}\right)
\end{array}\right] .
\end{aligned}
$$

The covariance matrix of $\mathbf{V}_{r}\left(l \cdot T_{s}\right)$ can be written as

$$
\begin{aligned}
& \mathbf{C}_{\mathbf{V}_{r}\left(l \cdot T_{s}\right) \mathbf{V}_{r}\left(l \cdot T_{s}\right)} \\
& =\left[\begin{array}{cc}
\sigma_{V_{I, r}\left(l \cdot T_{s}\right)}^{2} & C_{V_{I, r} V_{Q, r}}\left(l \cdot T_{s}, l \cdot T_{s}\right) \\
C_{V_{I, r} V_{Q, r}}\left(l \cdot T_{s}, l \cdot T_{s}\right) & \sigma_{V_{Q, r}}^{2}\left(l \cdot T_{s}\right)
\end{array}\right] .
\end{aligned}
$$


If we now assume that the received power is large, and that the coherence time of the source is much smaller than the integration time of the matched filters, then the central limit theorem applies, such that the output signals of the matched filters can be considered as jointly Gaussian. To be able to derive the (conditional) probability density function of the output signal of a four-way phase diversity receiver, it is useful to decorrelate the elements of $\mathbf{V}_{r}\left(l \cdot T_{s}\right)$, which means that we write them as a linear combination of two independent Gaussian variables $V_{I, r}^{\prime}\left(l \cdot T_{s}\right)$ and $V_{Q, r}^{\prime}\left(l \cdot T_{s}\right)$. If we write the latter two as a vector $\mathbf{V}_{r}^{\prime}\left(l \cdot T_{s}\right)$, then we can write

$$
\mathbf{V}_{r}\left(l \cdot T_{s}\right)=\mathbf{A}_{r, l} \mathbf{V}_{r}^{\prime}\left(l \cdot T_{s}\right)
$$

where $\mathbf{A}_{r, l}$ is a unitary matrix with columns that correspond

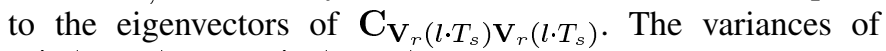
$V_{I, r}^{\prime}\left(l \cdot T_{s}\right)$ and $V_{Q, r}^{\prime}\left(l \cdot T_{s}\right)$ are equal to the eigenvalues of $\mathbf{C}_{\mathbf{V}_{r}\left(l \cdot T_{s}\right) \mathbf{V}_{r}\left(l \cdot T_{s}\right)}$, which are given by

$$
\begin{aligned}
\lambda_{1,2}= & \frac{R_{p d}^{2} P_{\mathrm{in}}^{2} G^{2} T_{s} \tau_{c}}{128 L_{X}^{2}} \\
& \cdot\left[4 \sum_{i_{1}=1}^{N} \sum_{i_{2}=1}^{N} P_{\bmod , i_{1}}\left(l \cdot T_{s}\right) P_{\bmod , i_{2}}\left(l \cdot T_{s}\right)\right. \\
& \left.+2 \sum_{i=1}^{N} P_{\bmod , i}^{2}\left(l \cdot T_{s}\right) \pm P_{\bmod , r}^{2}\left(l \cdot T_{s}\right)\right] \\
& +\frac{R_{p d} P_{\mathrm{in}} e \cdot G^{2} T_{s}}{4 L_{X}} \sum_{i=1}^{N} P_{\bmod , i}\left(l \cdot T_{s}\right) \\
& +S_{\mathrm{th}} G^{2} T_{s}
\end{aligned}
$$

and the corresponding eigenvectors are given by

$$
\begin{aligned}
& \mathbf{v}_{1}=\left[\begin{array}{l}
\cos \left(\Delta \phi_{r}+\phi_{\bmod , r}\left(l \cdot T_{s}\right)-\frac{3 \pi}{4}\right) \\
\cos \left(\Delta \phi_{r}+\phi_{\bmod , r}\left(l \cdot T_{s}\right)+\frac{3 \pi}{4}\right)
\end{array}\right] \\
& \mathbf{v}_{2}=\left[\begin{array}{c}
\cos \left(\Delta \phi_{r}+\phi_{\bmod , r}\left(l \cdot T_{s}\right)-\frac{\pi}{4}\right) \\
\cos \left(\Delta \phi_{r}+\phi_{\bmod , r}\left(l \cdot T_{s}\right)-\frac{3 \pi}{4}\right)
\end{array}\right] .
\end{aligned}
$$

Hence, the expected value of $\mathbf{V}_{r}^{\prime}\left(l \cdot T_{s}\right)$ is given by

$$
\begin{aligned}
\mathrm{E}\left[\mathbf{V}_{r}^{\prime}\left(l \cdot T_{s}\right)\right] & =\mathrm{E}\left[\mathbf{A}_{r, l}^{-1} \mathbf{V}_{r}\left(l \cdot T_{s}\right)\right] \\
& =\mathbf{A}_{r, l}^{\mathrm{T}} \mathrm{E}\left[\mathbf{V}_{r}\left(l \cdot T_{s}\right)\right] \\
& =\frac{R_{p d} P_{\text {in }} G \cdot T_{s}}{8 L_{X}} P_{\bmod , r}\left(l \cdot T_{s}\right) \cdot\left[\begin{array}{l}
1 \\
0
\end{array}\right]
\end{aligned}
$$

\section{Four-Way OOK Receiver}

For the four-way OOK receiver, the output signal can be written as

$$
\begin{aligned}
V_{\text {out }, r}\left(l \cdot T_{s}\right) & =V_{I, r}^{2}\left(l \cdot T_{s}\right)+V_{Q, r}^{2}\left(l \cdot T_{s}\right) \\
& =\mathbf{V}_{r}^{\mathrm{T}}\left(l \cdot T_{s}\right) \mathbf{V}_{r}\left(l \cdot T_{s}\right) \\
& =\left(\mathbf{V}_{r}^{\prime}\left(l \cdot T_{s}\right)\right)^{\mathrm{T}} \mathbf{A}_{r, l}^{\mathrm{T}} \mathbf{A}_{r, l} \mathbf{V}_{r}^{\prime}\left(l \cdot T_{s}\right) \\
& =\left(\mathbf{V}_{r}^{\prime}\left(l \cdot T_{s}\right)\right)^{\mathrm{T}} \mathbf{V}_{r}^{\prime}\left(l \cdot T_{s}\right) \\
& =\left(V_{I, r}^{\prime}\left(l \cdot T_{s}\right)\right)^{2}+\left(V_{Q, r}^{\prime}\left(l \cdot T_{s}\right)\right)^{2}
\end{aligned}
$$

since $\mathbf{A}_{r, l}$ is unitary. $V_{I, r}^{\prime}\left(l \cdot T_{s}\right)$ and $V_{Q, r}^{\prime}\left(l \cdot T_{s}\right)$ are Gaussian distributed and independent. Since OOK modulation is used, we have $\phi_{\bmod , i}(t)=0$ for all $i$. Using (43), and taking into account that the value of any $P_{\bmod , i}\left(l \cdot T_{s}\right)$ is always either 0 or 1 , the expected values and variances of $V_{I, r}^{\prime}\left(l \cdot T_{s}\right)$ and $V_{Q, r}^{\prime}\left(l \cdot T_{s}\right)$ can be written as

$$
\begin{aligned}
\mathrm{E}\left[V_{I, r}^{\prime}\left(l \cdot T_{s}\right)\right]= & \frac{R_{p d} P_{\mathrm{in}} G \cdot T_{s}}{8 L_{X}} P_{\mathrm{mod}, r}\left(l \cdot T_{s}\right) \\
\mathrm{E}\left[V_{Q, r}^{\prime}\left(l \cdot T_{s}\right)\right]= & 0 \\
\sigma_{V_{I, r}^{\prime}\left(l \cdot T_{s}\right)}^{2}= & \frac{R_{p d}^{2} P_{\mathrm{in}}^{2} G^{2} T_{s} \tau_{c}}{128 L_{X}^{2}} \\
& \cdot\left[4 N_{1}^{2}+2 N_{1}\left(1+4 P_{\bmod , r}\left(l \cdot T_{s}\right)\right)\right. \\
& \left.+7 P_{\bmod , r}\left(l \cdot T_{s}\right)\right]+\frac{R_{p d} P_{\mathrm{in}} e \cdot G^{2} T_{s}}{4 L_{X}} \\
& \cdot\left[N_{1}+P_{\bmod , r}\left(l \cdot T_{s}\right)\right]+S_{\mathrm{th}} G^{2} T_{s} \\
\sigma_{V_{Q, r}^{\prime}\left(l \cdot T_{s}\right)}^{2}= & \frac{R_{p d}^{2} P_{\mathrm{in}}^{2} G^{2} T_{s} \tau_{c}}{128 L_{X}^{2}} \\
& \cdot\left[4 N_{1}^{2}+2 N_{1}\left(1+4 P_{\bmod , r}\left(l \cdot T_{s}\right)\right)\right. \\
& \left.+5 P_{\bmod , r}\left(l \cdot T_{s}\right)\right]+\frac{R_{p d} P_{\mathrm{in}} e \cdot G^{2} T_{s}}{4 L_{X}} \\
& \cdot\left[N_{1}+P_{\bmod , r}\left(l \cdot T_{s}\right)\right]+S_{\mathrm{th}} G^{2} T_{s} .
\end{aligned}
$$

When a binary zero is transmitted, we have $\mathrm{E}\left[V_{I, r}^{\prime}\left(l \cdot T_{s}\right)\right]=$ $\mathrm{E}\left[V_{Q, r}^{\prime}\left(l \cdot T_{s}\right)\right]=0$ and $\sigma_{V_{I, r}^{\prime}\left(l \cdot T_{s}\right)}=\sigma_{V_{I, r}^{\prime}\left(l \cdot T_{s}\right)}$, so the conditional probability distribution of $V_{\text {out }, r}\left(l \cdot T_{s}\right)$ for $P_{\bmod , r}(l \cdot$ $\left.T_{s}\right)=0$ and $N_{1}=n$ is central $\chi^{2}$-distributed with two degrees of freedom

$$
\begin{aligned}
& f_{V_{\mathrm{out}, r}\left(l \cdot T_{s}\right) \mid P_{\mathrm{mod}, r}\left(l \cdot T_{s}\right)=0, N_{1}=n}(v) \\
& =\left.\frac{1}{2 \sigma_{V_{I, r}^{\prime}\left(l \cdot T_{s}\right)}^{2}} \exp \left(-\frac{v}{2 \sigma_{V_{I, r}^{\prime}\left(l \cdot T_{s}\right)}^{2}}\right)\right|_{P_{\mathrm{mod}, r}\left(l \cdot T_{s}\right)=0, N_{1}=n}
\end{aligned}
$$

for $v>0$. When a binary one is transmitted, the portion of the probability density that is of interest to us can be approximated by neglecting $V_{Q, r}^{\prime}\left(l \cdot T_{s}\right)$, resulting in a noncentral $\chi^{2}$-distribution with one degree of freedom

$$
\begin{aligned}
& f_{V_{\text {out }, r}\left(l \cdot T_{s}\right) \mid P_{\mathrm{mod}, r}\left(l \cdot T_{s}\right)=1, N_{1}=n}(v) \\
& \approx \frac{1}{\sigma_{V_{I, r}^{\prime}\left(l \cdot T_{s}\right)} \sqrt{2 \pi v}} \exp \left(-\frac{v+\mathrm{E}^{2}\left[V_{I, r}^{\prime}\left(l \cdot T_{s}\right)\right]}{2 \sigma_{V_{I, r}^{\prime}\left(l \cdot T_{s}\right)}^{2}}\right) \\
& \left.\quad \cdot \cosh \left(\frac{\sqrt{v} \mathrm{E}\left[V_{I, r}^{\prime}\left(l \cdot T_{s}\right)\right]}{\sigma_{V_{I, r}^{\prime}\left(l \cdot T_{s}\right)}^{2}}\right)\right|_{P_{\mathrm{mod}, r}\left(l \cdot T_{s}\right)=1, N_{1}=n} .
\end{aligned}
$$

The optimum detection threshold corresponds to the point of intersection of (67) and (68) [19]. It can be verified that this can be approximated by

$$
\begin{aligned}
V_{\mathrm{opt}} & \approx \frac{1}{4} \mathrm{E}^{2}\left[V_{I, r}^{\prime}\left(l \cdot T_{s}\right) \mid P_{\mathrm{mod}, r}\left(l \cdot T_{s}\right)=1\right] \\
& =\frac{R_{p d}^{2} P_{\mathrm{in}}^{2} G^{2} T_{s}^{2}}{256 L_{X}^{2}} .
\end{aligned}
$$


Using (45), we find that the total probability of bit error is given by

$$
\begin{aligned}
P_{e}= & \left(\frac{1}{2}\right)^{N} \sum_{n=0}^{N-1}\left(\begin{array}{c}
N-1 \\
n
\end{array}\right) \\
& \cdot\left[\int_{V_{\mathrm{opt}}}^{\infty} f_{V_{\mathrm{out}, r}\left(l \cdot T_{s}\right) \mid P_{\mathrm{mod}, r}\left(l \cdot T_{s}\right)=0, N_{1}=n}(v) d v\right. \\
& \left.+\int_{-\infty}^{V_{\mathrm{opt}}} f_{V_{\mathrm{out}, r}\left(l \cdot T_{s}\right) \mid P_{\mathrm{mod}, r}\left(l \cdot T_{s}\right)=1, N_{1}=n}(v) d v\right] \\
\approx & \left(\frac{1}{2}\right)^{N} \sum_{n=0}^{N-1}\left(\begin{array}{c}
N-1 \\
n
\end{array}\right) \\
& \cdot\left[\exp \left(-\frac{\gamma_{0}(n)}{4}\right)+Q\left(\sqrt{\frac{\gamma_{1}(n)}{2}}\right)\right]
\end{aligned}
$$

where

$$
\begin{aligned}
\gamma_{0}(n)= & \frac{\mathrm{E}^{2}\left[V_{I, r}^{\prime}\left(l \cdot T_{s}\right) \mid P_{\mathrm{mod}, r}\left(l \cdot T_{s}\right)=1\right]}{2 \sigma_{V_{I, r}^{\prime}\left(l \cdot T_{s}\right) \mid P_{\mathrm{mod}, r}\left(l \cdot T_{s}\right)=0, N_{1}=n}^{2}} \\
= & R_{p d}^{2} P_{\mathrm{in}}^{2} T_{s}\left(R_{p d}^{2} P_{\mathrm{in}}^{2} \tau_{c}\left(4 n^{2}+2 n\right)\right. \\
& \left.+32 e \cdot R_{p d} P_{\mathrm{in}} L_{X} \cdot n+128 S_{\mathrm{th}} L_{X}^{2}\right)^{-1} \\
\gamma_{1}(n)= & \frac{\mathrm{E}^{2}\left[V_{I, r}^{\prime}\left(l \cdot T_{s}\right) \mid P_{\mathrm{mod}, r}\left(l \cdot T_{s}\right)=1\right]}{2 \sigma_{V_{I, r}^{\prime}}^{2}\left(l \cdot T_{s}\right) \mid P_{\mathrm{mod}, r}\left(l \cdot T_{s}\right)=1, N_{1}=n} \\
= & R_{p d}^{2} P_{\mathrm{in}}^{2} T_{s}\left(R_{p d}^{2} P_{\mathrm{in}}^{2} \tau_{c}\left(4 n^{2}+10 n+7\right)\right. \\
& +32 e \cdot R_{p d} P_{\mathrm{in}} L_{X}(n+1) \\
& \left.+128 S_{\mathrm{th}} L_{X}^{2}\right)^{-1}
\end{aligned}
$$

and the Gaussian tail probability $Q(x)$ is defined as

$$
Q(x) \triangleq \frac{1}{\sqrt{2 \pi}} \int_{x}^{\infty} \exp \left(-\frac{z^{2}}{2}\right) d z .
$$

A similar analysis shows that the bit error rate of a phase-synchronized balanced two-way OOK detector is given by

$$
P_{e} \approx\left(\frac{1}{2}\right)^{N} \sum_{n=0}^{N-1}\left(\begin{array}{c}
N-1 \\
n
\end{array}\right)\left[Q\left(\sqrt{\frac{\gamma_{0}(n)}{2}}\right)+Q\left(\sqrt{\frac{\gamma_{1}(n)}{2}}\right)\right]
$$

with

$$
\begin{aligned}
\gamma_{0}(n)=R_{p d}^{2} P_{\mathrm{in}}^{2} T_{s} & \left(R_{p d}^{2} P_{\mathrm{in}}^{2} \tau_{c}\left(4 n^{2}+2 n\right)\right. \\
& \left.+16 e \cdot R_{p d} P_{\mathrm{in}} L_{X} n+32 S_{\mathrm{th}} L_{X}^{2}\right)^{-1} \\
\gamma_{1}(n)=R_{p d}^{2} P_{\mathrm{in}}^{2} T_{s}( & R_{p d}^{2} P_{\mathrm{in}}^{2} \tau_{c}\left(4 n^{2}+10 n+7\right) \\
& \left.+16 e \cdot R_{p d} P_{\mathrm{in}} L_{X}(n+1)+32 S_{\mathrm{th}} L_{X}^{2}\right)^{-1}
\end{aligned}
$$

Obviously, the four-way OOK phase diversity receiver requires over $3 \mathrm{~dB}$ more optical power to achieve the same performance as the two-way balanced OOK receiver, because the optical power is divided over twice as many photo detectors. Even when the received power is very large, however (such that source-induced noise becomes dominant), the two-way balanced receiver will perform slightly better than the four-way phase diversity receiver. (The result for the two-way receiver then corresponds to the BER that is derived in [21].) The difference in performance can be quantified by defining the bandwidth efficiency $\eta$ as the ratio of the total network capacity $N \cdot R_{\mathrm{b}}=N / T_{s}$ and the source bandwidths $\Delta f$ at which the probability of bit error goes to $10^{-9}$ for high received powers

$$
\left.\eta \triangleq \frac{N \cdot R_{\mathrm{b}}}{\Delta f}\right|_{P_{e}=10^{-9}, P_{\mathrm{in}} \rightarrow \infty}
$$

In Fig. 7, this is plotted as a function of the number of channels, in which Gaussian source spectra are assumed, such that (6) holds. Apparently, four-way phase diversity detection introduces only a small degradation in bandwidth efficiency when OOK modulation is used. In Section V-F, the performance of different receivers will be compared in a numerical example of a coherence multiplexed network.

\section{Four-Way DPSK Receiver}

The differential output signals of the four-way DPSK phase diversity receiver can be written as

$$
\begin{aligned}
& V_{\mathrm{DI}, r}\left(l \cdot T_{s}\right)=V_{I, r}\left(l \cdot T_{s}\right) V_{I, r}\left((l-1) T_{s}\right) \\
& +V_{Q, r}\left(l \cdot T_{s}\right) V_{Q, r}\left((l-1) T_{s}\right) \\
& =\mathbf{V}_{r}^{\mathrm{T}}\left(l \cdot T_{s}\right) \mathbf{V}_{r}\left((l-1) T_{s}\right) \\
& =\left(\mathbf{V}_{r}^{\prime}\left(l \cdot T_{s}\right)\right)^{\mathrm{T}} \mathbf{A}_{r, l}^{\mathrm{T}} \mathbf{A}_{r, l-1} \mathbf{V}_{r}^{\prime}\left((l-1) T_{s}\right) \\
& =\left[V_{I, r}^{\prime}\left(l \cdot T_{s}\right) V_{I, r}^{\prime}\left((l-1) T_{s}\right)\right. \\
& \left.+V_{Q, r}^{\prime}\left(l \cdot T_{s}\right) V_{Q, r}^{\prime}\left((l-1) T_{s}\right)\right] \\
& \cdot \cos \left(\phi_{\bmod , r}\left(l \cdot T_{s}\right)-\phi_{\bmod , r}\left((l-1) T_{s}\right)\right) \\
& +\left[V_{I, r}^{\prime}\left(l \cdot T_{s}\right) V_{Q, r}^{\prime}\left((l-1) T_{s}\right)\right. \\
& \left.-V_{Q, r}^{\prime}\left(l \cdot T_{s}\right) V_{I, r}^{\prime}\left((l-1) T_{s}\right)\right] \\
& \cdot \sin \left(\phi_{\bmod , r}\left(l \cdot T_{s}\right)\right. \\
& \left.-\phi_{\bmod , r}\left((l-1) T_{s}\right)\right) \\
& V_{\mathrm{DQ}, r}\left(l \cdot T_{s}\right)=V_{Q, r}\left(l \cdot T_{s}\right) V_{I, r}\left((l-1) T_{s}\right) \\
& -V_{I, r}\left(l \cdot T_{s}\right) V_{Q, r}\left((l-1) T_{s}\right) \\
& =\mathbf{V}_{r}^{\mathrm{T}}\left(l \cdot T_{s}\right)\left[\begin{array}{cc}
0 & -1 \\
1 & 0
\end{array}\right] \mathbf{V}_{r}\left((l-1) T_{s}\right) \\
& =\left(\mathbf{V}_{r}^{\prime}\left(l \cdot T_{s}\right)\right)^{\mathrm{T}} \mathbf{A}_{r, l}^{\mathrm{T}}\left[\begin{array}{cc}
0 & -1 \\
1 & 0
\end{array}\right] \\
& \text { - } \mathbf{A}_{r, l-1} \mathbf{V}_{r}^{\prime}\left((l-1) T_{s}\right) \\
& =\left[V_{I, r}^{\prime}\left(l \cdot T_{s}\right) V_{I, r}^{\prime}\left((l-1) T_{s}\right)\right. \\
& \left.+V_{Q, r}^{\prime}\left(l \cdot T_{s}\right) V_{Q, r}^{\prime}\left((l-1) T_{s}\right)\right] \\
& \cdot \sin \left(\phi_{\bmod , r}\left(l \cdot T_{s}\right)-\phi_{\bmod , r}\left((l-1) T_{s}\right)\right) \\
& +\left[V_{Q, r}^{\prime}\left(l \cdot T_{s}\right) V_{I, r}^{\prime}\left((l-1) T_{s}\right)\right. \\
& \left.-V_{I, r}^{\prime}\left(l \cdot T_{s}\right) V_{Q, r}^{\prime}\left((l-1) T_{s}\right)\right] \\
& \cdot \cos \left(\phi_{\bmod , r}\left(l \cdot T_{s}\right)\right. \\
& \left.-\phi_{\bmod , r}\left((l-1) T_{s}\right)\right)
\end{aligned}
$$

where $V_{I, r}^{\prime}\left(l \cdot T_{s}\right), V_{Q, r}^{\prime}\left(l \cdot T_{s}\right), V_{I, r}^{\prime}\left((l-1) T_{s}\right)$ and $V_{Q, r}^{\prime}((l-$ 1) $\left.T_{s}\right)$ are Gaussian and independent. Since DPSK modulation 


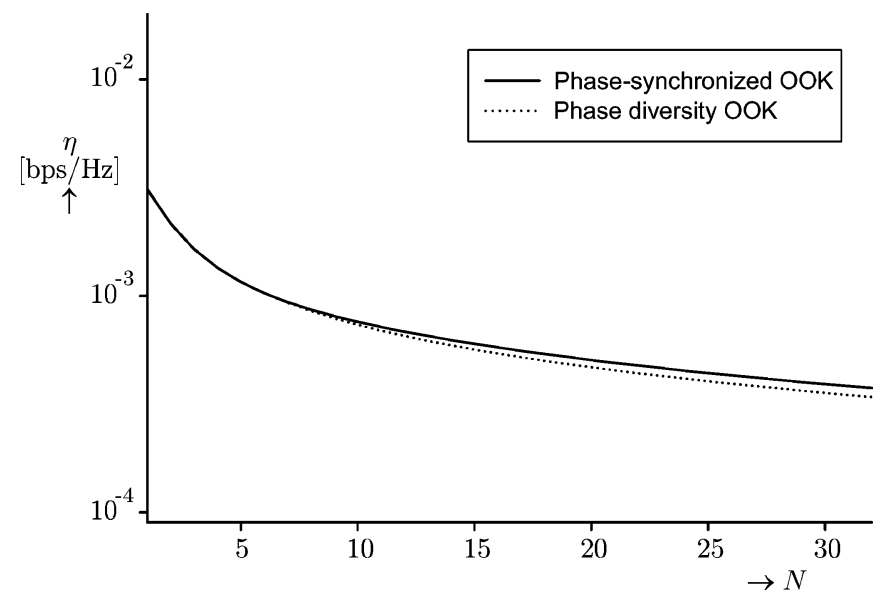

Fig. 7. Maximum bandwidth efficiency $\eta$ as a function of the number of channels $N$ for OOK modulation.

is used, we have $P_{\bmod , i}(t)=1$ for all $i$. The expected values and variances of $V_{I, r}^{\prime}\left(l \cdot T_{s}\right)$ and $V_{Q, r}^{\prime}\left(l \cdot T_{s}\right)$ can now be written as

$$
\begin{aligned}
\mathrm{E}\left[V_{I, r}^{\prime}\left(l \cdot T_{s}\right)\right]= & \frac{R_{p d} P_{\mathrm{in}} G \cdot T_{s}}{8 L_{X}} \\
\mathrm{E}\left[V_{Q, r}^{\prime}\left(l \cdot T_{s}\right)\right]= & 0 \\
\sigma_{V_{I, r}^{\prime}\left(l \cdot T_{s}\right)}^{2}= & \frac{R_{p d}^{2} P_{\mathrm{in}}^{2} G^{2} T_{s} \tau_{c}}{128 L_{X}^{2}}\left(4 N^{2}+2 N+1\right) \\
& +\frac{R_{p d} P_{\mathrm{in}} e \cdot G^{2} T_{s}}{4 L_{X}} N+S_{\mathrm{th}} G^{2} T_{s} \\
\sigma_{V_{Q, r}^{\prime}\left(l \cdot T_{s}\right)}^{2}= & \frac{R_{p d}^{2} P_{\mathrm{in}}^{2} G^{2} T_{s} \tau_{c}}{128 L_{X}^{2}}\left(4 N^{2}+2 N-1\right) \\
& +\frac{R_{p d} P_{\mathrm{in}} e \cdot G^{2} T_{s}}{4 L_{X}} N+S_{\mathrm{th}} G^{2} T_{s} .
\end{aligned}
$$

Finding an exact analytical expression for the resulting bit error probability using this decorrelation is impossible, since $\sigma_{V_{Q, r}^{\prime}\left(l \cdot T_{s}\right)}^{2} \neq \sigma_{V_{I, r}^{\prime}\left(l \cdot T_{s}\right)}^{2}$. For $N>1$, however, we can approximate $\sigma_{V_{Q, r}^{\prime}\left(l \cdot T_{s}\right)}^{2} \approx \sigma_{V_{I, r}^{\prime}\left(l \cdot T_{s}\right)}^{2}$, such that the classical results for DPSK in AWGN (for example, from [19]) can be used as a close upper bound, resulting in

$$
P_{e, B D} \approx \frac{1}{2} \exp \left(-\gamma_{s}\right)
$$

for binary DPSK

$$
P_{e, Q D} \approx \frac{1}{2} \exp \left(-\gamma_{s}\right)\left[I_{0}\left(\frac{\gamma_{s}}{\sqrt{2}}\right)+2 \sum_{n=1}^{\infty}(\sqrt{2}-1)^{n} I_{n}\left(\frac{\gamma_{s}}{\sqrt{2}}\right)\right]
$$

for DQPSK, and

$$
P_{e, M D} \approx \frac{2}{k} Q\left(\sqrt{\gamma_{s}} \sin \left(\frac{\pi}{M}\right)\right)
$$

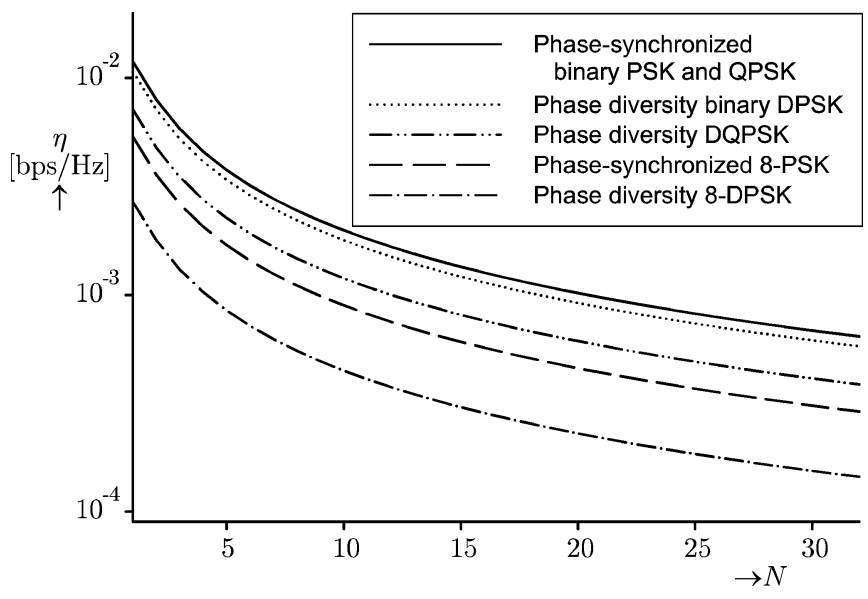

Fig. 8. Maximum bandwidth efficiency $\eta$ as a function of the number of channels $N$ for (D)PSK modulation.

for $M$-ary DPSK with $M \geq 8$, where the SNR per symbol $\gamma_{s}$ is given by

$$
\begin{aligned}
\gamma_{s}= & \frac{\mathrm{E}^{2}\left[V_{I, r}^{\prime}\left(l \cdot T_{s}\right)\right]}{2 \sigma_{V_{I, r}^{\prime}\left(l \cdot T_{s}\right)}^{2}} \\
= & R_{p d}^{2} P_{\mathrm{in}}^{2} L_{X}^{2} T_{s}\left(R_{p d}^{2} P_{\mathrm{in}}^{2} \tau_{c}\left(4 N^{2}+2 N+1\right)\right. \\
& \left.+32 e \cdot R_{p d} P_{\mathrm{in}} L_{X} N+128 S_{\mathrm{th}} L_{X}^{2}\right)^{-1}
\end{aligned}
$$

and $I_{n}(x)$ corresponds to the modified Bessel function of the first kind and order $n$

$$
I_{n}(x) \triangleq \frac{1}{2 \pi} \int_{0}^{2 \pi} \exp (x \cos \theta-n \theta) d \theta
$$

and $k$ is the number of bits per symbol, so $M=2^{k}$.

It is interesting to compare the performance of the DPSK phase diversity receiver with the performance of a phase-synchronized PSK receiver. For QPSK and $M$-ary PSK, a similar receiver structure should be used; the only difference is that the delay-and-multiply circuit is omitted and a phase-synchronization circuit is added. The bit error probabilities can be shown to be

$$
P_{e, Q} \approx Q\left(\sqrt{\gamma_{s}}\right)
$$

for QPSK and

$$
P_{e, M} \approx \frac{2}{k} Q\left(\sqrt{2 \gamma_{s}} \sin \left(\frac{\pi}{M}\right)\right)
$$

for $M$-ary PSK with $M \geq 8$, where $\gamma_{s}$ is given by (87). For binary PSK, a two-way balanced receiver can be used (see Section II). It can be shown that its bit error probability is given by

$$
P_{e, B} \approx Q\left(\sqrt{2 \gamma_{s}}\right)
$$

with

$$
\begin{aligned}
\gamma_{s}=R_{p d}^{2} P_{\mathrm{in}}^{2} L_{X}^{2} T_{s} & \left(R_{p d}^{2} P_{\mathrm{in}}^{2} \tau_{c}\left(4 N^{2}+2 N+1\right)\right. \\
& \left.+16 e \cdot R_{p d} P_{\mathrm{in}} L_{X} N+32 S_{\mathrm{th}} L_{X}^{2}\right)^{-1}
\end{aligned}
$$


Obviously, the four-way binary DPSK phase diversity receiver requires over $3 \mathrm{~dB}$ more optical power in order to achieve the same performance as the phase-synchronized binary PSK two-way balanced receiver, just like in the case for OOK modulation. The performance for high received power can be compared by once more observing the bandwidth efficiencies of the different receiver types; this is illustrated in Fig. 8. (Note that in the case of $M$-ary PSK, the bit rate is given by $R_{\mathrm{b}}=k / T_{s}$.) Apparently, phase diversity detection of binary DPSK modulated signals results in only a small degradation in bandwidth efficiency with respect to phase-synchronized detection of binary PSK modulated signals. As long as the effect of chromatic dispersion is neglected, the bandwidth efficiency decreases when $M$ increases. Moreover, binary (D)PSK results in a higher bandwidth efficiency than OOK. We will come back to the performance comparison in the numerical example in Section V-F.

\section{E. Three-Way OOK Receiver}

Using a similar procedure as in the case of four-way phase diversity, we can write the balanced signals at the sampling instants as a vector $\mathbf{V}_{r}\left(l \cdot T_{s}\right)=\left[V_{\mathrm{a}, r}\left(l \cdot T_{s}\right), V_{\mathrm{b}, r}\left(l \cdot T_{s}\right), V_{c, r}(l \cdot\right.$ $\left.\left.T_{s}\right)\right]^{\mathrm{T}}$, with expected value

$$
\begin{aligned}
\mathrm{E}\left[\mathbf{V}_{r}\left(l \cdot T_{s}\right)\right]= & \frac{R_{p d} P_{\mathrm{in}} G \cdot T_{s}}{4 \sqrt{3} L_{X}} P_{\bmod , r}\left(l \cdot T_{s}\right) \\
& \cdot\left[\begin{array}{c}
\cos \left(\Delta \phi_{r}+\phi_{\bmod , r}\left(l \cdot T_{s}\right)+\frac{2 \pi}{3}\right) \\
\cos \left(\Delta \phi_{r}+\phi_{\bmod , r}\left(l \cdot T_{s}\right)\right) \\
\cos \left(\Delta \phi_{r}+\phi_{\bmod , r}\left(l \cdot T_{s}\right)-\frac{2 \pi}{3}\right)
\end{array}\right] .
\end{aligned}
$$

The covariance matrix of $\mathbf{V}_{r}\left(l \cdot T_{s}\right)$ depends on the implementation form of the three-way receiver, as amplification can be performed either prior to or after balancing. These situations can be analyzed simultaneously, however, by denoting the power spectral density of the output voltage of an eventual TIA before balancing by $S_{\text {th,pre }}$ and an eventual TIA after balancing by $S_{\text {th,post }}$. (So $S_{\text {th,pre }}=0$ if amplification is performed after balancing and $S_{\text {th,post }}=0$ if amplification is performed prior to balancing.)

For $N>1$, the first element of the covariance matrix $\mathbf{C}_{\mathbf{V}_{r}\left(l \cdot T_{s}\right) \mathbf{V}_{r}\left(l \cdot T_{s}\right)}$ can be closely approximated by

$$
\begin{aligned}
\sigma_{V_{\mathrm{a}, r}\left(l \cdot T_{s}\right)}^{2}= & \frac{R_{p d}^{2} P_{\mathrm{in}}^{2} G^{2} T_{s} \tau_{c}}{96 L_{X}^{2}} \\
& \cdot\left[4 \sum_{i_{1}=1}^{N} \sum_{i_{2}=1}^{N} P_{\bmod , i_{1}}\left(l \cdot T_{s}\right) P_{\mathrm{mod}, i_{2}}\left(l \cdot T_{s}\right)\right. \\
& +2 \sum_{i=1}^{N} P_{\bmod , i}^{2}\left(l \cdot T_{s}\right)+P_{\bmod , r}^{2}\left(l \cdot T_{s}\right) \\
& \left.\cdot \cos \left(2 \Delta \phi_{r}+2 \phi_{\bmod , r}\left(l \cdot T_{s}\right)-\frac{2 \pi}{3}\right)\right] \\
+ & \frac{R_{p d} P_{\mathrm{in}} e \cdot G^{2} T_{s}}{3 L_{X}} \sum_{i=1}^{N} P_{\bmod , i}\left(l \cdot T_{s}\right) \\
+ & \left(2 S_{\mathrm{th}, \operatorname{pre}}+S_{\mathrm{th}, \operatorname{post}}\right) G^{2} T_{s}
\end{aligned}
$$

and similar expressions can be found for the remaining elements. Again, the elements of $\mathbf{V}_{r}\left(l \cdot T_{s}\right)$ are jointly Gaussian, and can be decorrelated by writing them as linear combinations of independent Gaussian variables $V_{I, r}^{\prime}\left(l \cdot T_{s}\right), V_{Q, r}^{\prime}\left(l \cdot T_{s}\right)$ and $V_{X, r}^{\prime}\left(l \cdot T_{s}\right)$. The latter three can be written as a vector $\mathbf{V}_{r}^{\prime}\left(l \cdot T_{s}\right)$, such that we can write

$$
\mathbf{V}_{r}\left(l \cdot T_{s}\right)=\mathbf{A}_{r, l} \mathbf{V}_{r}^{\prime}\left(l \cdot T_{s}\right)
$$

where the columns of $\mathbf{A}_{r, l}$ correspond to the eigenvectors of $\mathbf{C}_{\mathbf{V}_{r}\left(l \cdot T_{s}\right) \mathbf{V}_{r}\left(l \cdot T_{s}\right)}$. It can be proven that the eigenvalues of $\mathbf{C}_{\mathbf{V}_{r}\left(l \cdot T_{s}\right) \mathbf{V}_{r}\left(l \cdot T_{s}\right)}$ are now given by

$$
\begin{aligned}
\lambda_{1,2}= & \frac{R_{p d}^{2} P_{\mathrm{in}}^{2} G^{2} T_{s} \tau_{c}}{64 L_{X}^{2}} \\
& \cdot\left[4 \sum_{i_{1}=1}^{N} \sum_{i_{2}=1}^{N} P_{\mathrm{mod}, i_{1}}\left(l \cdot T_{s}\right) P_{\mathrm{mod}, i_{2}}\left(l \cdot T_{s}\right)\right. \\
& \left.+2 \sum_{i=1}^{N} P_{\mathrm{mod}, i}^{2}\left(l \cdot T_{s}\right) \pm P_{\mathrm{mod}, r}^{2}\left(l \cdot T_{s}\right)\right] \\
& +\frac{R_{p d} P_{\mathrm{in}} e \cdot G^{2} T_{s}}{2 L_{X}} \sum_{i=1}^{N} P_{\mathrm{mod}, i}\left(l \cdot T_{s}\right) \\
& +\left(3 S_{\mathrm{th}, \mathrm{pre}}+S_{\mathrm{th}, \mathrm{post}}\right) G^{2} T_{s} \\
\lambda_{3}= & S_{\mathrm{th}, \mathrm{post}} G^{2} T_{s}
\end{aligned}
$$

with corresponding (normalized) eigenvectors

$$
\begin{aligned}
& \mathbf{v}_{1}=\sqrt{\frac{2}{3}}\left[\begin{array}{c}
\cos \left(\Delta \phi_{r}+\phi_{\bmod , r}\left(l \cdot T_{s}\right)+\frac{2 \pi}{3}\right) \\
\cos \left(\Delta \phi_{r}+\phi_{\bmod , r}\left(l \cdot T_{s}\right)\right) \\
\cos \left(\Delta \phi_{r}+\phi_{\bmod , r}\left(l \cdot T_{s}\right)-\frac{2 \pi}{3}\right)
\end{array}\right] \\
& \mathbf{v}_{2}=\sqrt{\frac{2}{3}}\left[\begin{array}{c}
\cos \left(\Delta \phi_{r}+\phi_{\bmod , r}\left(l \cdot T_{s}\right)+\frac{\pi}{6}\right) \\
\cos \left(\Delta \phi_{r}+\phi_{\bmod , r}\left(l \cdot T_{s}\right)-\frac{\pi}{2}\right) \\
\cos \left(\Delta \phi_{r}+\phi_{\bmod , r}\left(l \cdot T_{s}\right)+\frac{5 \pi}{6}\right)
\end{array}\right] \\
& \mathbf{v}_{3}=\frac{1}{\sqrt{3}}\left[\begin{array}{c}
1 \\
1 \\
1
\end{array}\right] .
\end{aligned}
$$

Hence, the expected value of $\mathbf{V}_{r}^{\prime}\left(l \cdot T_{s}\right)$ is given by

$$
\begin{aligned}
\mathrm{E}\left[\mathbf{V}_{r}^{\prime}\left(l \cdot T_{s}\right)\right] & =\mathrm{E}\left[\mathbf{A}_{r, l}^{-1} \mathbf{V}_{r}\left(l \cdot T_{s}\right)\right] \\
& =\mathbf{A}_{r, l}^{\mathrm{T}} \mathrm{E}\left[\mathbf{V}_{r}\left(l \cdot T_{s}\right)\right] \\
& =\frac{R_{p d} P_{\mathrm{in}} G \cdot T_{s}}{4 \sqrt{2} L_{X}} P_{\bmod , r}\left(l \cdot T_{s}\right) \cdot\left[\begin{array}{l}
1 \\
0 \\
0
\end{array}\right] .
\end{aligned}
$$

The output samples of the three-way OOK receiver can be written as

$$
V_{\text {out }, r}\left(l \cdot T_{s}\right)=\left(V_{I, r}^{\prime}\left(l \cdot T_{s}\right)\right)^{2}+\left(V_{Q, r}^{\prime}\left(l \cdot T_{s}\right)\right)^{2}+\left(V_{X, r}^{\prime}\left(l \cdot T_{s}\right)\right)^{2} \text {. }
$$

When amplification is performed prior to balancing, we have $S_{\text {th,post }}=0$ and $S_{\text {th,pre }}=S_{\mathrm{th}}$, and hence, both the expected value and the variance of $V_{X, r}^{\prime}\left(l \cdot T_{s}\right)$ are zero, so we simply have $V_{X, r}^{\prime}\left(l \cdot T_{s}\right)=0$. (102) then reduces to (62), so it can be 
reasoned that the BER for the three-way phase diversity receiver is then given by (70), with $\gamma_{0}(n)$ and $\gamma_{1}(n)$ given by

$$
\begin{aligned}
\gamma_{0}(n)=R_{p d}^{2} P_{\mathrm{in}}^{2} T_{s}\left(R_{p d}^{2} P_{\mathrm{in}}^{2} \tau_{c}\left(4 n^{2}+2 n\right)\right. \\
\left.+32 e \cdot R_{p d} P_{\mathrm{in}} L_{X} n+192 S_{\mathrm{th}} L_{X}^{2}\right)^{-1}
\end{aligned}
$$

and

$$
\begin{aligned}
\gamma_{1}(n)= & R_{p d}^{2} P_{\mathrm{in}}^{2} T_{s}\left(R_{p d}^{2} P_{\mathrm{in}}^{2} \tau_{c}\left(4 n^{2}+10 n+7\right)\right. \\
& \left.+32 e \cdot R_{p d} P_{\mathrm{in}} L_{X}(n+1)+192 S_{\mathrm{th}} L_{X}^{2}\right)^{-1} .
\end{aligned}
$$

When amplification is performed after balancing, however, we have $S_{\mathrm{th} \text {,pre }}=0$ and $S_{\mathrm{th} \text {,post }}=S_{\mathrm{th}}$. When a binary zero is transmitted in that case, we have $\mathrm{E}\left[V_{I, r}^{\prime}\left(l \cdot T_{s}\right)\right]=\mathrm{E}\left[V_{I, r}^{\prime}(l \cdot\right.$ $\left.\left.T_{s}\right)\right]=\mathrm{E}\left[V_{X, r}^{\prime}\left(l \cdot T_{s}\right)\right]=0$ and $\sigma_{V_{I, r}^{\prime}\left(l \cdot T_{s}\right)}=\sigma_{V_{Q, r}^{\prime}\left(l \cdot T_{s}\right)} \neq$ $\sigma_{V_{X, r}^{\prime}\left(l \cdot T_{s}\right)}$. Hence, the conditional probability density function of the output signal can be found by convolving a central $\chi^{2}$-distribution with two degrees of freedom and a central $\chi^{2}$-distribution with one degree of freedom, resulting in

$$
\begin{aligned}
& f_{V_{\mathrm{out}, r}\left(l \cdot T_{s}\right) \mid P_{\mathrm{mod}, r}\left(l \cdot T_{s}\right)=0, N_{1}=n}(v) \\
& =\frac{1}{\sigma_{V_{I, r}^{\prime}\left(l \cdot T_{s}\right)} \sqrt{\sigma_{V_{I, r}^{\prime}\left(l \cdot T_{s}\right)}^{2}-\sigma_{V_{X, r}^{\prime}}^{2}\left(l \cdot T_{s}\right)}} \\
& \cdot\left[\frac{1}{2}-Q\left(\sqrt{\frac{v}{\sigma_{V_{X, r}^{\prime}\left(l \cdot T_{s}\right)}^{2}}-\frac{v}{\sigma_{V_{I, r}^{\prime}\left(l \cdot T_{s}\right)}^{2}}}\right)\right] \\
& \left.\cdot \exp \left(-\frac{v}{2 \sigma_{V_{I, r}^{\prime}\left(l \cdot T_{s}\right)}^{2}}\right)\right|_{P_{\mathrm{mod}, r}\left(l \cdot T_{s}\right)=0, N_{1}=n}
\end{aligned}
$$

for $v>0$. For a binary one, a similar approximation can be made as in the case of the four-way receiver; see (68). Performing a similar analysis as for the four-way receiver results in the following probability of bit error

$$
\begin{aligned}
P_{e} \approx Q & \left(\sqrt{\frac{\gamma_{X}}{2}}\right)+\left(\frac{1}{2}\right)^{N} \sum_{n=0}^{N-1}\left(\begin{array}{c}
N-1 \\
n
\end{array}\right) \\
& \cdot\left[\sqrt{\frac{\gamma_{X}}{\gamma_{X}-\gamma_{0}(n)}} \exp \left(-\frac{\gamma_{0}(n)}{4}\right)\right. \\
& \cdot\left(1-2 Q\left(\sqrt{\frac{\gamma_{X}-\gamma_{0}(n)}{2}}\right)\right) \\
& \left.+Q\left(\sqrt{\frac{\gamma_{1}(n)}{2}}\right)\right]
\end{aligned}
$$

where

$$
\begin{aligned}
\gamma_{0}(n)=R_{p d}^{2} P_{\mathrm{in}}^{2} T_{s}( & R_{p d}^{2} P_{\mathrm{in}}^{2} \tau_{c}\left(4 n^{2}+2 n\right) \\
& \left.+32 e \cdot R_{p d} P_{\mathrm{in}} L_{X} \cdot n+64 S_{\mathrm{th}} L_{X}^{2}\right)^{-1}
\end{aligned}
$$

$$
\begin{gathered}
\gamma_{1}(n)=R_{p d}^{2} P_{\mathrm{in}}^{2} T_{s}\left(R_{p d}^{2} P_{\mathrm{in}}^{2} \tau_{c}\left(4 n^{2}+10 n+7\right)\right. \\
+32 e \cdot R_{p d} P_{\mathrm{in}} L_{X}(n+1) \\
\left.+64 S_{\mathrm{th}} L_{X}^{2}\right)^{-1} \\
\gamma_{X}=\frac{R_{p d}^{2} P_{\mathrm{in}}^{2} T_{s}}{64 S_{\mathrm{th}} L_{X}^{2}} .
\end{gathered}
$$

(Note that this is the same as (70) when $S_{\text {th }}=0$.) By comparing these expressions to the corresponding expressions that were found for the four-way phase diversity receiver, one can conclude the following:

- When the thermal receiver noise can be neglected with respect to source-induced noise and shot noise, the performance of the three-way and four-way OOK phase diversity receivers are the same. (Hence, they have the same bandwidth efficiency; see Fig. 7.)

- When the thermal receiver noise is dominating, the three-way OOK phase diversity receiver with amplification prior to balancing requires approximately $0.9 \mathrm{~dB}$ more optical power than the four-way OOK phase diversity receiver in order to achieve the same performance (provided that the same amplifiers are used in both cases). The three-way OOK receiver with amplification after balancing requires $1.5 \mathrm{~dB}$ less than the four-way $\mathrm{OOK}$ receiver, however.

Note, however, that balancing prior to amplification is not straightforward in a three-way receiver, as balancing by means of a triangular configuration of the photodiodes conflicts with the necessity of simultaneously operating the photodiodes in the reversed-bias mode.

\section{F. Numerical Example of a Coherence Multiplexed Network}

To make a realistic comparison between the different modulation formats and receiver structures in which the limited received power is incorporated, a numerical example of a coherence multiplexed optical communication system is considered, in which 16 transmitters are connected to 16 receivers by means of a 16-port combiner, a common fiber and a 16-port splitter.

The light sources (for example, SLDs) are assumed to operate around $1310 \mathrm{~nm}$ (to minimize chromatic dispersion) and have a linewidth of $40 \mathrm{~nm}$. Assuming a Gaussian spectral profile, the coherence time $\tau_{c}$ can be calculated using (6), resulting in $\tau_{c} \approx 0.1 \mathrm{ps}$. One can verify that chromatic dispersion in standard single-mode fiber can then indeed be neglected for bit rate-length products up to $1 \mathrm{~Gb} / \mathrm{s} \cdot \mathrm{km} \mathrm{[20].} \mathrm{It} \mathrm{is} \mathrm{assumed} \mathrm{that}$ each light source couples $10 \mathrm{dBm}$ of optical power into the optical circuit.

As an example we assume that the MZI's are fabricated as planar optical waveguide devices in silicon oxynitride (SiON) technology [22]. To limit crosstalk between the users, the minimum path imbalance and the spacing between the path imbalances should be larger than the coherence time. Using (4), (10), and (14), it can be proven that crosstalk between the users is below $-80 \mathrm{~dB}$ if we choose

$$
T_{\mathrm{Tx}, i}=3 i \cdot \tau_{c}=i \cdot 0.3 \mathrm{ps} .
$$

Fabrication inaccuracies in SiON waveguides lead to typical worst case errors in the effective index that are given by [22]

$$
\frac{\Delta N_{\mathrm{eff}}}{N_{\mathrm{eff}}} \approx 5 \cdot 10^{-4} .
$$

This results in small delay mismatches in a matched transmitterreceiver pair. The largest mismatch occurs for the pair with the largest path imbalance and is given by

$$
\left|T_{\mathrm{Rx}, r}-T_{\mathrm{Tx}, r}\right|_{\max } \approx 2 \frac{\Delta N_{\mathrm{eff}}}{N_{\mathrm{eff}}} T_{\mathrm{Tx}, 16} \approx 5 \text { fs. }
$$




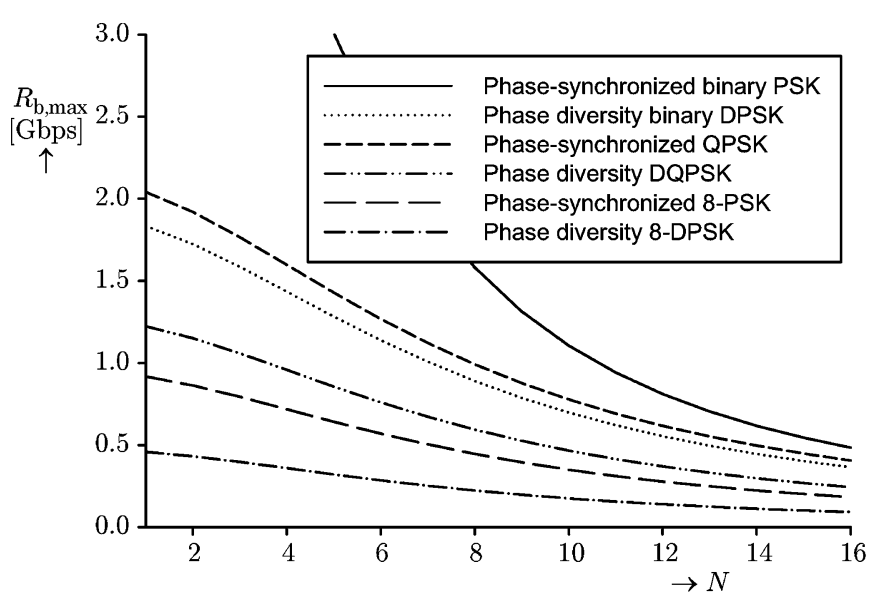

(a)

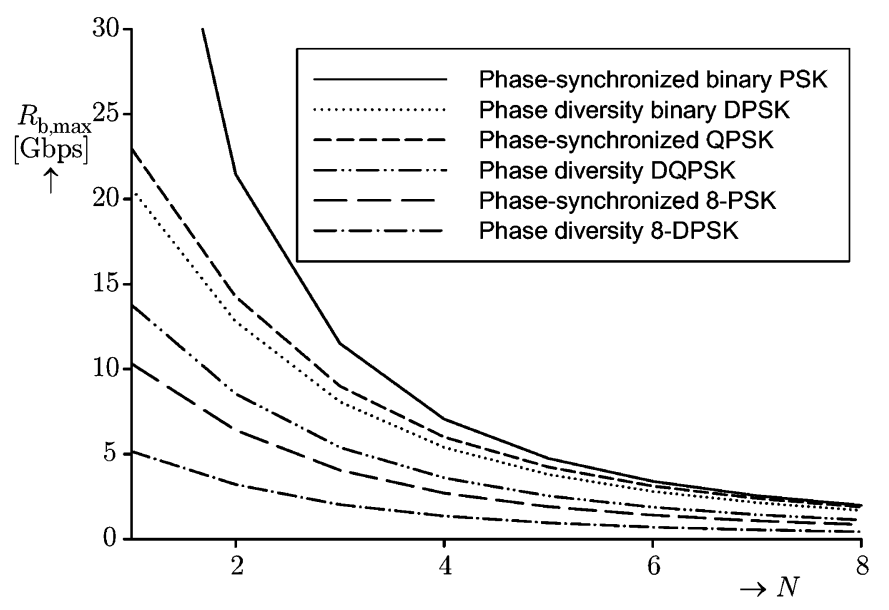

(c)

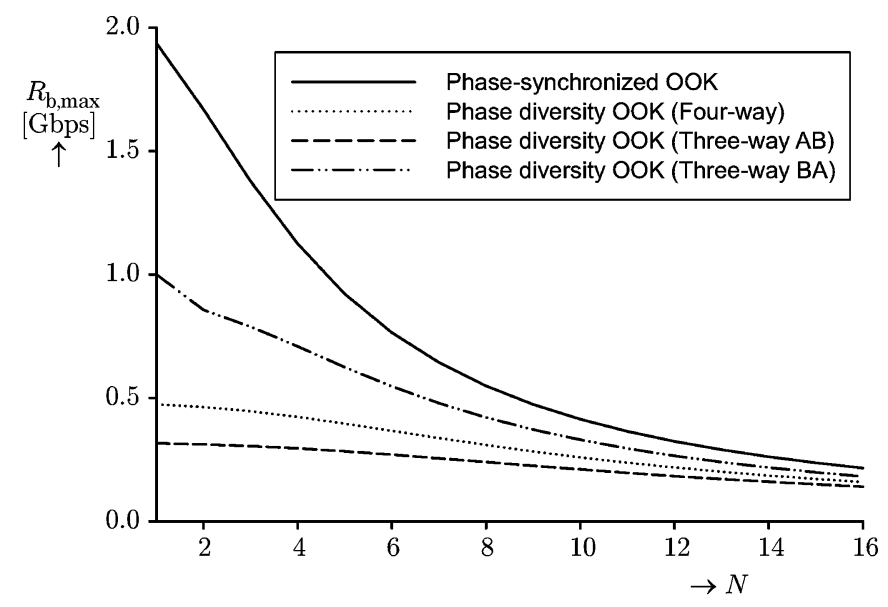

(b)

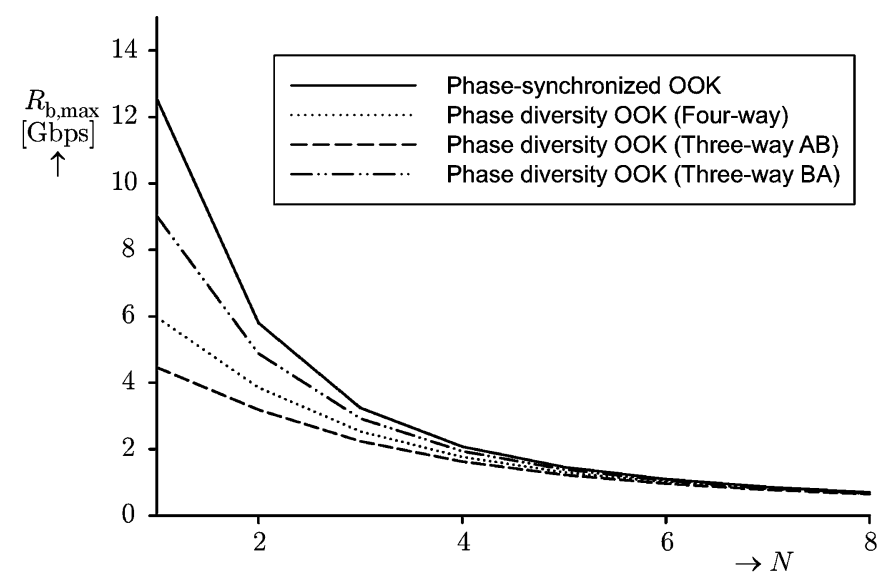

(d)

Fig. 9. Maximum bit rate per channel that can be achieved at a BER of $10^{-9}$ as a function of the number of active channels $N$ (AB=amplification prior to balancing, BA=amplification after balancing). (a) 16 transmitters and receivers using (D)PSK modulation. (b) 16 transmitters and receivers using OOK modulation. (c) 8 transmitters and receivers using (D)PSK modulation. (d) 8 transmitters and receivers using OOK modulation.

Using (4) and (11) it follows that the attenuation of a desired channel at the output of a phase diversity receiver due to coherence mismatching is smaller than $0.1 \mathrm{~dB}$. The corresponding phase mismatch follows from

$$
\Delta \phi_{\max }=2 \pi f_{c}\left|T_{\mathrm{Rx}, r}-T_{\mathrm{Tx}, r}\right|_{\max } \approx 7 \mathrm{rad}
$$

These two results show that typical fabrication inaccuracies do result in significant phase mismatches but do not result in significant coherence mismatches. This actually motivates the use of phase diversity in this numerical example.

It is assumed that the total excess loss in the optical circuits and chip-fiber couplings is approximately $3 \mathrm{~dB}$. Together with the intrinsic splitting loss of $12 \mathrm{~dB}$ in both the splitter and the combiner, this results in $L_{X} \approx 512$.

In the receivers, the photodiodes are assumed to have responsivities $R_{p d}=0.8 \mathrm{~A} / \mathrm{W}$. Since optical receivers for bit rates up to a few Gbps have been realized in practice with measured equivalent input noise currents in the order of $7 \mathrm{pA} / \sqrt{\mathrm{Hz}}$, we assume that $S_{\mathrm{th}} \approx 5 \cdot 10^{-23} \mathrm{~A}^{2} / \mathrm{Hz}$.

With this numerical data, we use the formulas derived in the previous subsection to find the maximum bit rate per channel that can be achieved at a BER of $10^{-9}$ as a function of the number of transmitters $N$ that are actually active (hence causing a corresponding amount of source-induced noise and shot noise in each receiver), for the different modulation formats and receiver structures. The results are presented in Fig. 9(a) for (D)PSK modulation, and Fig. 9(b) for OOK modulation.

Apparently, binary PSK results in a superior performance compared to the other modulation formats. Obviously, the significant difference between phase-synchronized detection of binary PSK and phase diversity detection of binary DPSK is mainly caused by the fact that in a phase diversity receiver, the power is divided over four photo detectors rather than two, because Fig. 8 shows that the source-induced noise limited bit rates for phase-synchronized detection of binary PSK and phase diversity detection of binary DPSK are nearly the same.

A similar reasoning holds for the difference in maximum bit rate for phase-synchronized detection and phase diversity detection of OOK. The difference between the three considered phase diversity configurations for OOK can be explained as follows: in the case of amplification after balancing, the optical 
power has to be divided over the most photodiodes in case of the four-way receiver, hence resulting in the worst performance of these two receivers. When, in the three-way receiver, amplification is performing prior to balancing however, then the noise current of the amplifier affects two of the balanced signals rather than only one, which increases the effect of thermal noise on the output signal of the receiver.

In Fig. 9(c) and (d) the same results are shown for a coherence multiplexed network with eight transmitters and receivers. (Hence, we have $L_{X} \approx 128$.) Especially for a low number of active channels $N$, the $6 \mathrm{dBm}$ increase in received optical power significantly boosts the bit rates that can be achieved per channel, indicating that thermal noise is the dominating noise when the number of active channels is low.

When $N$ increases, however, we see that the achievable bit rate per channel decreases faster in the 8-transmitter network than in the 16-transmitter network. This illustrates the relatively strong presence of thermal noise in the 16-transmitter network, since the signal-to-thermal noise ratio does not depend on the number of channels, whereas the signal-to-source induced noise ratio and the signal-to-shot noise ratio do.

\section{CONCLUSION}

It has been proven analytically that the output signal of a coherence multiplex receiver can be stabilized by means of a phase diversity network. The advantage of this configuration is that neither an external feedback loop nor a frequency shifter is required, resulting in less complicated -and hence potentially less expensive- receiver units. Performance figures for several receiver types (two-way balanced detection and three-way and four-way balanced phase diversity detection) and several modulation formats (OOK, PSK and DPSK) were derived analytically, showing that phase diversity detection introduces only a minor performance degradation with respect to two-way balanced detection, as long as the received optical power is large such that thermal receiver noise is negligible. However, numerical examples with networks consisting of 16 and 8 transmission units, respectively, have shown that the inherent increase in splitting loss in a phase diversity coupler significantly decreases the detector performance due to thermal receiver noise. Binary (D)PSK modulation results in the best performance, provided that the effect of chromatic fiber dispersion is negligible. The performances of the three-way and four-way phase diversity receivers are similar, as long as the noise in the receivers is dominated either by source-induced noise or shot noise. When thermal receiver noise dominates, however, the performance of the three-way receiver differs from the performance of the four-way receiver, depending on the order of the balancing operation and amplification. If balancing were performed prior to amplification, the three-way receiver would require approximately $1.5 \mathrm{~dB}$ less optical power than the four-way receiver in order to obtain the same performance. Proper balancing of the photodiodes in the three-way receiver would be achieved in a much easier way, however, if the order of balancing and amplification were reversed. Unfortunately, this would boost the required optical power by up to $2.4 \mathrm{~dB}$.

\section{ACKNOWLEDGMENT}

The authors would like to thank Dr. C. G. H. Roeloffzen for helpful discussions on integrated optics technology.

\section{REFERENCES}

[1] D. D. Sampson, G. J. Pendock, and R. A. Griffin, "Photonic code-division multiple-access communications," Fiber Integrat. Opt., vol. 16, pp. 129-157, 1997.

[2] J. W. Goodman, Statistical Optics. New York: Wiley, 1985.

[3] R. H. Wentworth, "Theoretical noise performance of coherence-multiplexed interferometric sensors," J. Lightwave Technol., vol. 7, pp. 941-956, June 1989.

[4] G. J. Pendock and D. D. Sampson, "Increasing the transmission capacity of coherence multiplexed communication systems by using differential detection," IEEE Photon. Technol. Lett., vol. 7, pp. 1504-1506, Dec. 1995.

[5] J. L. Brooks, R. H. Wentworth, R. C. Youngquist, M. Tur, B. Y. Kim, and H. J. Shaw, "Coherence multiplexing of fiber-optic interferometric sensors," J. Lightwave Technol., vol. 3, pp. 1062-1072, Oct. 1985.

[6] J.-P. Goedgebuer and A. Hamel, "Coherence multiplexing using a parallel array of electrooptic modulators and multimode semiconductor lasers," IEEE J. Quantum Electron., vol. 23, pp. 2224-2237, Dec. 1987.

[7] K. W. Chu and F. M. Dickey, "Optical coherence multiplexing for interprocessor communications," Opt. Eng., vol. 30, pp. 337-344, Mar. 1991.

[8] A. Meijerink, R. O. Taniman, G. H. L. M. Heideman, and W. van Etten, "Comparison of three coherence multiplex system topologies," in Proc. 8th IEEE/LEOS Symp. Benelux, Enschede, The Netherlands, Nov. 2003, pp. 141-144.

[9] R. A. Griffin, D. D. Sampson, and D. A. Jackson, "Demonstration of data-transmission using coherent correlation to reconstruct a coded pulse sequence," IEEE Photon. Technol. Lett., vol. 4, pp. 513-515, May 1992.

[10] D. A. Blair and G. D. Cormack, "Optimal source linewidth in a coherence multiplexed optical fiber communications system," J. Lightwave Technol., vol. 10, pp. 804-810, June 1992.

[11] A. W. Davis, M. J. Pettitt, J. P. King, and S. Wright, "Phase diversity techniques for coherent optical receivers," J. Lightwave Technol., vol. 5, pp. 561-572, Apr. 1987.

[12] P. Healey, "Dimensioning an optical-fiber spread-spectrum multiple-access communication system," Opt. Lett., vol. 12, pp. 425-427, June 1987.

[13] W. van Etten and A. Meijerink, "Optical stabilization of coherence multiplex output signal by means of a phase diversity network," in Proc. 6th IEEE/LEOS Symp. Benelux, Brussels, Belgium, Dec. 2001, pp. 149-152.

[14] A. Meijerink, G. H. L. M. Heideman, and W. van Etten, "Performance evaluation of an OOK coherence multiplex receiver based on $4 \times 4$ phase diversity detection," in Proc. XXVIIth URSI G.A. (CDROM), Maastricht, The Netherlands, Aug. 2002, paper D7.O.6.

[15] J. M. Heaton and M. J. Jenkins, "General matrix theory of self-imaging in multimode interference (MMI) couplers," IEEE Photon. Technol. Lett., vol. 11, pp. 212-214, Feb. 1999.

[16] R. G. Priest, "Analysis of fiber interferometer utilizing $3 \times 3$ fiber coupler," IEEE J. Quantum Electron., vol. 18, pp. 1601-1603, Oct. 1982

[17] A. Meijerink, G. H. L. M. Heideman, and W. van Etten, "BER analysis of a DPSK phase diversity receiver for coherence multiplexing," in Proc. 23rd Symp. Inform. Theory Benelux, Louvain-la-Neuve, Belgium, May 2002, pp. 269-276.

[18] - " $M$-ary (D)PSK modulation in coherence multiplex systems," in Proc. 7th IEEE/LEOS Symp. Benelux, Amsterdam, The Netherlands, Dec. 2002, pp. 207-210.

[19] J. G. Proakis, Digital Communications, Singapore: McGraw-Hill, 1983

[20] A. Meijerink, N. Niëns, G. H. L. M. Heideman, and W. van Etten, "Chromatic fiber dispersion in single-mode coherence multiplex systems," in Proc. 10th IEEE/CVT Symp. Benelux (CDROM), Eindhoven, The Netherlands, Nov. 2003, paper 9.

[21] G. J. Pendock and D. D. Sampson, "Capacity of coherence-multiplexed CDMA networks," Opt. Commun., vol. 143, pp. 109-117, Nov. 1997.

[22] K. Wörhoff, P. V. Lambeck, and A. Driessen, "Design, tolerance analysis, and fabrication of silicon oxynitride based planar optical waveguides for communication devices," J. Lightwave Technol., vol. 17, pp. 1401-1407, Aug. 1999. 


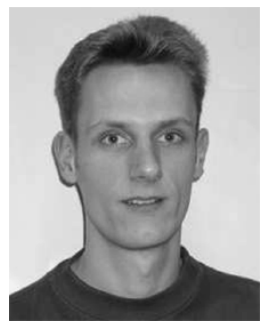

Arjan Meijerink ( $\left.\mathrm{S}^{\prime} 00\right)$ was born in Almelo, The Netherlands, in 1976. He received the M.Sc. (Hons.) degree in electrical engineering from the University of Twente, Enschede, The Netherlands, in January 2001. He is currently working toward the Ph.D. degree in the Telecommunication Engineering Group, University of Twente.

From January to May 2000, he was a guest at Ericsson Business Mobile Networks in Enschede, developing error concealment techniques for Bluetooth voice links. His current research interests include system-level design and performance analysis of optical communication systems based on coherence multiplexing.

Mr. Meijerink shared the Gauss Award for the best young researcher's presentation during the 22nd Symposium on Information and Communication Theory in the Benelux, in May 2001, and in July 2001, he was awarded the second prize in the IEEE Region 8 Student Paper Contest.

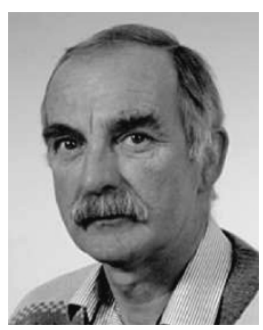

Geert H. L. M. Heideman was born in Oldenzaal, The Netherlands, in 1940. He received the M.Sc. degree in electrical engineering from Delft University of Technology, Delft, The Netherlands, in 1965.

In 1965, he was appointed Assistant Professor at the University of Twente, Enschede, The Netherlands. He was engaged in signal and information theory. In 1974, he became a Senior Scientist and, in 1986, he was appointed Associate Professor at the same university. He retired in 2003.

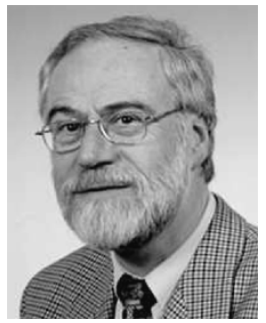

Wim van Etten (M'80-SM'91) was born in Zevenbergen, The Netherlands, in 1942 . He received the M.Sc. and Ph.D. degrees in electrical engineering from Eindhoven University of Technology, Eindhoven, The Netherlands, in 1969 and 1976, respectively.

From 1969 to 1970 , he was with Philips Electronics, developing circuits for oscilloscopes. In 1970, he became an Assistant Professor at Eindhoven University of Technology, Faculty of Electrical Engineering. From 1970 to 1976, he was engaged in research on the transmission of digital signals via coaxial and multiwire cables. Since 1976, he has been involved with research and education on optical fiber communications. In 1985, he was appointed Associate Professor at the Eindhoven University of Technology. In 1994, he became a Full Professor of Telecommunications at the University of Twente, Enschede, the Netherlands. His current interests comprise optical communications, mobile communications, detection, and simulation of communication systems. He is the author or coauthor of more than 60 papers in international journals, conferences, and symposia. He is first author of Fundamentals of Optical Fiber Communications (Englewood, NJ: Prentice-Hall, 1991.)

Prof. Van Etten is a member of the Dutch Institute of Electronic and Radio Engineers (NERG). He is chairman of the executive committee of the IEEE Benelux joint Chapter on Communications and Vehicular Technology. Two of his papers were selected for a collection of 57 key papers on communications that appeared in the last 50 years in IEEE ComSoc publications. He was a member of organizing committees and program committees of various conferences and symposia. 